\title{
Formal symplectic groupoid
}

\section{Journal Article}

\section{Author(s):}

Cattaneo, Alberto S.; Dherin, Benoit; Ferlder, G.

Publication date:

2005-02

Permanent link:

https://doi.org/10.3929/ethz-b-000033716

Rights / license:

$\underline{\text { In Copyright - Non-Commercial Use Permitted }}$

Originally published in:

Communications in Mathematical Physics 253(3), https://doi.org/10.1007/s00220-004-1199-z 


\title{
Formal Symplectic Groupoid
}

\author{
Alberto S. Cattaneo ${ }^{1, \star}$, Benoit Dherin ${ }^{2, \star \star}$, Giovanni Felder ${ }^{2, \star \star}$ \\ 1 Institut für Mathematik, Universität Zürich-Irchel, Winterthurerstrasse 190, 8057 Zürich, Switzerland. \\ E-mail: asc@math.unizh.ch \\ 2 D-MATH, ETH-Zentrum, 8092 Zürich, Switzerland. E-mail: dherin@math.ethz.ch; \\ felder@math.ethz.ch
}

Received: 5 January 2004 / Accepted: 16 April 2004

Published online: 20 October 2004 - () Springer-Verlag 2004

\begin{abstract}
The multiplicative structure of the trivial symplectic groupoid over $\mathbb{R}^{d}$ associated to the zero Poisson structure can be expressed in terms of a generating function. We address the problem of deforming such a generating function in the direction of a non-trivial Poisson structure so that the multiplication remains associative. We prove that such a deformation is unique under some reasonable conditions and we give the explicit formula for it. This formula turns out to be the semi-classical approximation of Kontsevich's deformation formula. For the case of a linear Poisson structure, the deformed generating function reduces exactly to the $\mathrm{CBH}$ formula of the associated Lie algebra. The methods used to prove existence are interesting in their own right as they come from an at first sight unrelated domain of mathematics: the Runge-Kutta theory of the numeric integration of ODE's.
\end{abstract}

\section{Introduction}

In this paper we give a formal version of the integration of Poisson manifolds by symplectic groupoids. The solution of this formal integration problem relies on the existence of a generating function for which we give here the explicit formula. This generating function turns out to be a universal Campbell-Baker-Hausdorff $(\mathrm{CBH})$ formula for the non-linear case. It reduces to the usual $\mathrm{CBH}$ formula when the Poisson structure comes from a Lie algebra. This generating function can be interpreted as the semi-classical part of the Kontsevich deformation quantization formula. This fact reminds of us the origin of symplectic groupoids which were first introduced by Weinstein in [6], Karasev in [11], and Zakrwewski in [18] as a tool to quantize the algebra of functions on a Poisson manifold. This section is devoted to recall some basic features of the program of quantization by symplectic groupoid, to formulate the formal integration problem for Poisson

* A.S.C. acknowledges partial support of SNF Grant No. 20-100029/1.

$\star \star$ B.D. and G.F. acknowledge partial support of SNF Grant No. 21-65213.01. 
manifolds and to state the main theorem of this article which gives a positive answer to the formal integration problem.

1.1. Quantization by symplectic groupoid. The program of quantization by symplectic groupoid is an attempt to quantize the algebra of functions on Poisson manifolds by geometric means.

It is based mainly on the belief or hope, coming from geometric quantization, that there should exist a kind of correspondence or dictionary between the world of symplectic manifold (classical level) and the world of linear spaces (quantum level). This correspondence, as explained in [1], is summarized in the following table:

\begin{tabular}{ll}
\hline Symplectic world & Linear world \\
\hline$M$ & $Q(M)$ \\
$L \subset M$ & $Q(L) \in Q(M)$ \\
$\bar{M}$ & $Q(\bar{M})=Q(M)^{*}$ \\
$Q(M \times N)$ & $Q(M) \otimes Q(N)$ \\
\hline
\end{tabular}

Here $M$ is a symplectic manifold, $\bar{M}$ the same manifold with opposite symplectic structure, $L$ a Lagrangian submanifold, and $Q(M)$ a complex vector space. $Q$ stands for the "Quantization functor". In particular, canonical relations, i.e., Lagrangian submanifolds of $\bar{M} \times N$ are sent by $Q$ to linear maps from $Q(M)$ to $Q(N)$. The main ingredient is the assumption that quantization is functorial, i.e., the composition of canonical relations should be sent to the composition of linear maps (see [16]). If such a quantization functor existed, we could ask the following question:

To what kind of symplectic manifold should we associate an algebra (i.e., a vector space with an associative product)?

Answering this question leads directly to the notion of symplectic groupoid, see [17].

Definition 1. A symplectic groupoid is a Lie groupoid $G$ (see [1] for a precise definition of a Lie groupoid) with a symplectic form $\omega$ for which the multiplication space $G^{(m)}=\{(x, y, x \bullet y) / x, y \in G$ are composable elements $\}$ is a Lagrangian submanifold of $\bar{G} \times \bar{G} \times G(\bar{G}$ being the symplectic manifold with symplectic form $-\omega)$. It can be shown (see [14]) that, given a symplectic groupoid $G$, there is an induced Poisson structure on the base space $G^{(0)}$. Conversely, given a Poisson manifold $P$ we call a symplectic groupoid over $P$ any symplectic groupoid $G$ such that the base space $G^{(0)}$ is diffeomorphic as a Poisson manifold to $P$. In this case we say that $G$ integrates $P$ and we call integrable Poisson manifolds the Poisson manifolds for which we can find such $a G$.

Applying the "Quantization functor" $Q$ to the symplectic groupoid $G$, we should then get a vector space $Q(G)$ and an associative product $Q\left(G^{(m)}\right)$ on it. The associativity of this product is guaranteed by the associativity of the groupoid multiplication and the functoriality of $Q$.

These facts suggest the following procedure to quantize Poisson manifolds $P$ :

Step 1 . Find a symplectic groupoid $G$ such that the base $G^{(0)}$ is diffeomorphic to the Poisson manifold $P$.

Step 2. Quantize (geometric quantization,...) $G$ and $G^{(m)}$ to get the quantum algebra. 
This is the idea of quantization by symplectic groupoid. Step 1 is known as the integrability problem and was recently completely settled. Coste, Dazord and Weinstein in [6] and independently Karasev in [11] showed the existence of a local symplectic groupoid over any Poisson manifold, "local" meaning that the multiplication is defined only on a neighborhood of the unit space. Cattaneo and Felder in [5] gave an explicit construction of a topological groupoid canonically associated to any Poisson manifold, which is a global symplectic groupoid whenever the Poisson structure is integrable. Crainic and Fernandes in [8] derived an if and only if criterium which tells one when the previous construction yields a manifold. Step 2 however was only partially achieved (see [15]).

If we compare this program with deformation quantization (see [2] and [13]), we see that starting with an integrable Poisson manifold $P$ whose symplectic groupoid is $G$ we should have the following relation between objects involved in these programs:

\begin{tabular}{lll}
\hline & Deformation quantization & Quantization by symplectic groupoids \\
\hline Semi-classical level & $?$ & $\left(G, G^{(m)}\right)$ \\
Quantum level & $\left(C^{\infty}(P)[[\epsilon]], *_{\epsilon}\right)$ & $\left(Q(G), Q\left(G^{(m)}\right)\right)$ \\
\hline
\end{tabular}

We can regard the symplectic groupoid over a Poisson manifold as a (semi-)classical version of the quantum algebra. In this picture $G^{(m)}$ should then correspond to a semiclassical version of the Kontsevich star-product formula. This is in some sense the case. Namely we can restate the integrability problem into a formal integration problem. The solution of this problem is called the formal symplectic groupoid over a Poisson manifold which is a formal version of the "true symplectic groupoid" that exists however even for non-integrable Poisson structures. This is exactly what the question marks stand for in the above table. Let us be more precise.

1.2. Formal integration problem for Poisson manifolds. In the sequel we will only consider Poisson structures $\alpha$ over $M=\mathbb{R}^{d}$. Suppose that $(M, \alpha)$ is integrable and that its symplectic groupoid $G$ satisfies the following two properties (which are always satisfied in a neighborhood of $M$ ):

(1) $G \subset T^{*} M \simeq \mathbb{R}^{* d} \times \mathbb{R}^{d}$

(2) $G^{(m)} \subset \overline{T^{*} M} \times \overline{T^{*} M} \times T^{*} M$ is an exact Lagrangian manifold, i.e., there exists a generating function $S: \mathbb{R}^{* d} \times \mathbb{R}^{* d} \times \mathbb{R}^{d} \rightarrow \mathbb{R}$ such that $G^{(m)}=\operatorname{graph}(d S)$.

We would like to see what sort of constraints the associativity of the groupoid product imposes on $S$. First of all we may remark that under the previous assumptions the product space $G^{(m)}$ can be described as follows

$$
G^{(m)}=\left\{\left(\left(p_{1}, \nabla_{p_{1}} S\right),\left(p_{2}, \nabla_{p_{2}} S\right),\left(\nabla_{x} S, x\right)\right):\left(p_{1}, p_{2}, x\right) \in B_{2}\right\},
$$

where the partial derivatives are evaluated at $\left(p_{1}, p_{2}, x\right) \in B_{2}:=\left(\mathbb{R}^{* d}\right)^{2} \times \mathbb{R}^{d}$.

The groupoid product associativity could be expressed by saying that, whenever the composition is allowed, we have $g=\bar{g} \bullet g_{3}$ and $g=g_{1} \bullet \tilde{g}$, where $\bar{g}=g_{1} \bullet g_{2}$ and $\tilde{g}=g_{2} \bullet g_{3}$. 
Denoting $g=(p, x), \bar{g}=(\bar{p}, \bar{x})$ and $\tilde{g}=(\tilde{p}, \tilde{x})$ implies that $\left(g_{1}, g_{2}, \bar{g}\right) \in$ $G^{(m)},\left(g_{2}, g_{3}, \tilde{g}\right) \in G^{(m)},\left(\bar{g}, g_{3}, g\right) \in G^{(m)}$ and $\left(g_{1}, \tilde{g}, g\right) \in G^{(m)}$. Now expressing $g_{1}, g_{2}, g_{3}, g, \bar{g}$ and $\tilde{g}$ each time in terms of the generating function $S$ and equating the different expressions found for the same element we get a system of six equations which can be summarized into the following more compact equation.

Symplectic Groupoid Associativity equation (SGA equation).

$$
S\left(p_{1}, p_{2}, \bar{x}\right)+S\left(\bar{p}, p_{3}, x\right)-\bar{x} \bar{p}=S\left(p_{2}, p_{3}, \tilde{x}\right)+S\left(p_{1}, \tilde{p}, x\right)-\tilde{x} \tilde{p},
$$

where

$$
\begin{array}{ll}
\bar{x}=\nabla_{p_{1}} S\left(\bar{p}, p_{3}, x\right), & \bar{p}=\nabla_{x} S\left(p_{1}, p_{2}, \bar{x}\right), \\
\tilde{x}=\nabla_{p_{2}} S\left(p_{1}, \tilde{p}, x\right), & \tilde{p}=\nabla_{x} S\left(p_{2}, p_{3}, \tilde{x}\right) .
\end{array}
$$

This equation encodes the associativity of the groupoid product into the generating function. It can also be seen from two other different points of view. First it is easy to check that one gets the SGA equation by requiring that the saddle point evaluation as $h$ goes to 0 of the two integrals

$$
\begin{gathered}
\int e^{\frac{i}{h}\left[S\left(p_{1}, p_{2}, x\right)+S\left(p, p_{3}, x\right)-p x\right]} \frac{d^{d} p d^{d} x}{(2 \pi h)^{d / 2}} \text { and } \\
\int e^{\frac{i}{h}\left[S\left(p_{2}, p_{3}, x\right)+S\left(p_{1}, p, x\right)-p x\right]} \frac{d^{d} p d^{d} x}{(2 \pi h)^{d / 2}}
\end{gathered}
$$

be equal. This allows us to provide in Sect. 7 a quick but non-rigorous proof of the existence of the generating function relying only on the associativity of the Kontsevich star product.

The second way to derive the SGA equation is symplectic reduction. Consider the symplectic groupoid $G$ over $M=\mathbb{R}^{d}$ as above. Let us call $\mathcal{L}_{S} \subset \bar{G} \times \bar{G} \times G$ the Lagrangian submanifold associated to the generating function $S$ (i.e., $\mathcal{L}_{S}=\operatorname{graph}(d S)$ ). Now consider the spaces $H(k)=\bar{G}^{k} \times G$ and the diagonal $\Delta_{l_{1}, \ldots, l_{k}} \subset \overline{H(k)} \times H\left(l_{1}\right) \times$ $\cdots \times H\left(l_{k}\right)$

$$
\Delta_{l_{1}, \ldots, l_{k}}=\left\{\left(g_{1}, \ldots, g_{k}, y\right),\left(x_{11}, \ldots, x_{1 l_{1}}, g_{1}\right), \ldots,\left(x_{k 1}, \ldots, x_{k l_{k}}, g_{k}\right)\right\} .
$$

This is a coisotropic subspace of $H(k) \times H\left(l_{1}\right) \times \cdots \times H\left(l_{k}\right)$. Then one can consider the symplectic reduction by the diagonal $\Delta_{l_{1}, \ldots, l_{k}}$ which sends Lagrangian submanifolds of $H(k) \times H\left(l_{1}\right) \times \cdots \times H\left(l_{k}\right)$ to Lagrangian submanifolds of $H\left(l_{1}+\cdots+l_{k}\right)$. In particular $\mathcal{L}_{S} \oplus \mathcal{L}_{S} \oplus \mathcal{L}_{I}(I(p, x)=p x)$ is sent to $\mathcal{L}_{1} \subset H(3)$ and $\mathcal{L}_{S} \oplus \mathcal{L}_{I} \oplus \mathcal{L}_{S}$ to $\mathcal{L}_{2} \subset H(3)$. One can check that $\mathcal{L}_{1}=\mathcal{L}_{2}$ iff $S$ satisfies the SGA equation. In fact we have here, hidden in the background, a structure of an operad, the Lagrangian operad (see [3]).

Now consider $M=\mathbb{R}^{d}$ with the zero Poisson structure. The symplectic groupoid $G_{0}$ over it is the cotangent bundle $\left(G_{0}=\mathbb{R}^{* d} \times \mathbb{R}^{d}\right)$. The source map and the target map $s, t: G_{0} \rightarrow \mathbb{R}^{d}$ are identified with the cotangent bundle projection. The inclusion $\epsilon: \mathbb{R}^{d} \rightarrow G_{0}$ is defined by $\epsilon(x)=(0, x)$, the inverse map $i: G_{0} \rightarrow G_{0}$ by $i(p, x)=$ $(-p, x)$ and the product is the fiber wise addition, i.e., $\left(p_{1}, x\right) \bullet\left(p_{2}, x\right)=\left(p_{1}+p_{2}, x\right)$. The product space $G_{0}^{(m)}$ can be seen as the graph of the differential of the function $S_{0}\left(p_{1}, p_{2}, x\right)=x\left(p_{1}+p_{2}\right)$. It is easy to check that $S_{0}$ satisfies the SGA equation. We investigate deformations of this trivial generating function. Let us be more precise. 
Definition 2. A deformation of the trivial generating function is a formal power series in $h, S_{h}=S_{0}+h S_{1}+h^{2} S_{2} \ldots$, obeying the $S G A$ equation and such that $S_{0}\left(p_{1}, p_{2}, x\right)=$ $x\left(p_{1}+p_{2}\right)$.

Such a deformation is called natural if

(1) $S_{n}(p, q, x)$ are polynomial in $q$ and $q$,

(2) $S_{n}(\lambda q, \lambda p, x)=\lambda^{n+1} S_{n}(p, q, x)$,

(3) $S_{n}(p, 0, x)=S_{n}(0, p, x)=0$,

(4) $S_{n}^{i}(p, p)=0$, where $S_{n}^{i}$ is the homogeneous part of $S_{n}$ of degree $i$ in the first argument.

In Sect. 2 we show that, provided we have a natural deformation $S_{h}=S_{0}+h S_{1}+$ $h^{2} S_{2}+\cdots$ of the trivial generating function, we can deform the structure maps of the trivial symplectic groupoid into

$$
\begin{aligned}
\epsilon_{h}(x) & =(0, x) \quad \text { unit map, } \\
i_{h}(p, x) & =(-p, x) \quad \text { inverse map, } \\
s_{h}(p, x) & =\nabla_{p_{2}} S_{h}(p, 0, x) \quad \text { source map, } \\
t_{h}(p, x) & =\nabla_{p_{1}} S_{h}(0, p, x) \quad \text { target map, }
\end{aligned}
$$

such that the groupoid structure is (formally) preserved.

Moreover there is a unique Poisson bracket on $\mathbb{R}^{d}$ such that the source, $s_{h}$, is a Poisson map with respect to the canonical symplectic structure on the formal symplectic groupoid. This Poisson bracket is given by $\{f, g\}_{\mathbb{R}^{d}}(x)=2 h S_{1}(d f, d g, x)$, the first order term of the generating function. We can now formulate the formal integration problem for Poisson manifolds.

Formal integration problem for Poisson manifolds. Given a Poisson structure on $\mathbb{R}^{d}$, does there exist a deformation of the trivial generating function such that the first order term is the original Poisson structure?

1.3. Main Result, main example, main interpretation. The following theorem gives a positive answer to the deformation problem for symplectic groupoids. This is the main result of this article.

Theorem 1. Given a Poisson structure $\alpha$ on $\mathbb{R}^{d}$ there exists a unique natural deformation of the trivial generating function such that the first order is precisely $\alpha$. Moreover we have an explicit formula for this deformation

$$
S_{h}\left(p_{1}, p_{2}, x\right)=x\left(p_{1}+p_{2}\right)+\sum_{n=1}^{\infty} \frac{h^{n}}{n !} \sum_{\Gamma \in T_{n, 2}} W_{\Gamma} \hat{B}_{\Gamma}\left(p_{1}, p_{2}, x\right),
$$

where $T_{n, 2}$ is the set of Kontsevich trees of type $(n, 2), W_{\Gamma}$ is the Kontsevich weight of $\Gamma$ and $\hat{B}_{\Gamma}$ is the symbol of the bidifferential operator $B_{\Gamma}$ associated to $\Gamma$.

Section 2 explains how to recover the structure maps from the deformed generating function. In Sect. 3 we present basic examples of formal symplectic groupoids. In particular the main one is in the case of a linear Poisson structure $\alpha^{i j}(x)=\alpha_{k}^{i j} x^{k}$, i.e., when one considers the Kirillov-Kostant Poisson structure on the dual $\mathcal{G}^{*}$ of a Lie algebra $\mathcal{G}$. 
In this case, the generating function of the symplectic groupoid over $\mathcal{G}^{*}$ reduces exactly to the Campbell-Baker-Hausdorff formula

$$
S_{h}\left(p_{1}, p_{2}, x\right)=\left\langle\frac{1}{h} C B H\left(h p_{1}, h p_{2}\right), x\right\rangle,
$$

where $\langle$,$\rangle is the natural pairing between \mathcal{G}$ and $\mathcal{G}^{*}$.

This basic example suggests to consider the generating function as a generalized $\mathrm{CBH}$ formula to the non-linear case and reproves in the linear case a result of V. Kathotia ([12]).

Sections 4 to 6 are devoted to the proof of Theorem 1. In Sect. 4 we introduce special graphs, the Cayley trees, which allow us to write down a perturbative version of the SGA equation. In Sect. 5 we describe the Kontsevich trees. We use them to produce an explicit solution for the deformation problem. Section 6 completes the proof of Theorem 1 .

In the last section we come to the comparison with deformation quantization. We see that the Kontsevich star-product can be put into the form

$$
f * g(x)=\left.\exp \left(\frac{1}{h} \sum_{i=0}^{\infty} h^{i} D_{i}\left(h \partial_{y}, h \partial_{z}, x\right)\right) f(y) g(z)\right|_{y=z=x},
$$

where

$$
S_{h}\left(p_{1}, p_{2}, x\right)=x\left(p_{1}+p_{2}\right)+\frac{1}{h} D_{0}\left(h p_{1}, h p_{2}, x\right) .
$$

This allows us to interpret the generating function as a semi-classical version of the Kontsevich star-product formula. At last, considering associativity of the star product of exponential functions, we are able to provide an elegant but non-rigorous proof of the existence part of Theorem 1.

1.4. Planned developments. One of the next objectives is to carry the construction of the formal symplectic groupoid to a general Poisson manifold. Karabegov in [10] already gave some hints on how to make such a globalisation. Namely, he constructed a global source and target provided there is a global star product on the Poisson manifold. These maps are proven to be Poisson maps whenever the Poisson manifold is symplectic.

A second possible development is to try to derive the existence of the deformation of the trivial generating function from a kind of "semi-classical" formality theorem.

At last we plan to compare the formal construction carried out in this article with the non-formal construction coming from the Poisson-sigma model (see [5]) and with the local symplectic groupoid construction of [6] and [11].

\section{Recovering the Formal Groupoid from the Generating Function}

In this section we show that one can recover formally the structure of symplectic groupoid from a generating function obeying the SGA equation.

Proposition 1. Let $S_{h}$ be a natural deformation of the trivial generating function which satisfies the SGA equation. Then the set $G_{h}=\mathbb{R}^{* d}[[h]] \times \mathbb{R}^{d}[[h]]$ can be given a structure of formal symplectic groupoid, i.e., the maps

$$
\begin{aligned}
\epsilon_{h}(x) & =(0, x) \quad \text { unitmap, } \\
i_{h}(p, x) & =(-p, x) \quad \text { inversemap, } \\
s_{h}(p, x) & =\nabla_{p_{2}} S_{h}(p, 0, x) \quad \text { sourcemap, } \\
t_{h}(p, x) & =\nabla_{p_{1}} S_{h}(0, p, x) \quad \text { targetmap, }
\end{aligned}
$$


and the multiplication given by

$$
G_{h}^{(m)}=\operatorname{graph}(d S)
$$

satisfy formally the axioms of a groupoid.

In particular, if we endow $G_{h}$ with the canonical symplectic form, then $G_{h}^{(m)}$ is formally Lagrangian in $\overline{G_{h}} \times \overline{G_{h}} \times G_{h}$.

Proof. The multiplication space being given by the graph of the differential of the generating function, we have automatically that the product, when defined, is associative (it satisfies the SGA equation) and that $G_{h}^{(m)}$ is formally a Lagrangian submanifold of $\overline{G_{h}} \times \overline{G_{h}} \times G$. We still have to check that the space of composable pairs is the right one, i.e., $(g, h) \in G_{h}^{(2)}$ iff $s(g)=t(h)$. We do that by noticing that all products are of the form $\left(p_{1}, \nabla_{p_{1}} S_{h}\left(p_{1}, p_{2}, x\right)\right) \bullet\left(p_{2}, \nabla_{p_{2}} S_{h}\left(p_{1}, p_{2}, x\right)\right)=\left(\nabla_{x} S_{h}\left(p_{1}, p_{2}, x\right), x\right)$. Thus the check amounts to see that

$$
s\left(p_{1}, \nabla_{p_{1}} S_{h}\left(p_{1}, p_{2}, x\right)\right)=t\left(p_{2}, \nabla_{p_{2}} S_{h}\left(p_{1}, p_{2}, x\right)\right)
$$

which can be seen by differentiating the SGA equation with respect to $p_{2}$, putting $p_{2}=0$ and using the fact that $S_{h}$ is natural.

It remains still to check the following axioms:

$$
\begin{aligned}
t(g h)=t(g) \quad(1), \quad s(g h)=s(h) \quad(2), \quad \epsilon(t(g)) g=g \quad(3), \quad g \epsilon(s(g))=g \\
s(i(g))=t(g) \quad(5), \quad i(g) g=\epsilon(s(g)) \quad(6), \quad g i(g)=\epsilon(t(g)) \quad(7) .
\end{aligned}
$$

Axiom 1 is obtained by differentiating the SGA equation w.r.t. $p_{1}$, putting $p_{1}=0$ and using naturality of $S_{h}$. Axiom 2 is similar but for replacing $p_{1}$ by $p_{3}$. Axiom 3 and Axiom 4 are direct consequences of the naturality. The last three axioms are however a bit more tricky. First let us prove two lemmas.

Lemma 1 (Inversion of source and target). Denote $F_{p}(x)=\nabla_{p_{2}} S_{h}(p, 0, x)$ and $G_{p}(x)=\nabla_{p_{1}} S_{h}(0, p, x)$. Then $F_{p}$ and $G_{p}$ are formal diffeomorphisms and their inverses are given by

$$
F_{p}^{-1}(x)=\nabla_{p_{2}} S_{h}(-p, p, x), \quad G_{p}^{-1}(x)=\nabla_{p_{1}} S_{h}(p,-p, x) .
$$

Proof. Denote $\bar{F}_{p}(x)=\nabla_{p_{2}} S_{h}(-p, p, x)$ and $\bar{G}_{p}(x)=\nabla_{p_{1}} S_{h}(p,-p, x)$. Differentiating the SGA equation w.r.t. $p_{1}$, putting $p_{1}=p, p_{2}=-p, p_{3}=p$, we get that $\bar{G}_{p} \circ G_{p}=i d$. Putting $p_{1}=0, p_{2}=p, p_{3}=-p$, we get $G_{p} \circ \bar{G}_{p}=i d$. Thus $\bar{G}_{p}=G_{p}^{-1}$. Similarly differentiating the SGA equation w.r.t. $p_{3}$, putting $p_{1}=p$, $p_{2}=-p, p_{3}=p$, we get that $\bar{F}_{p} \circ F_{p}=i d$. Putting $p_{1}=p, p_{2}=-p, p_{3}=0$, we get $F_{p} \circ \bar{F}_{p}=i d$. Thus $\bar{F}_{p}=F_{p}^{-1}$.

Lemma 2 (Relation between source and target). Denote $F_{p}(x)=\nabla_{p_{2}} S_{h}(p, 0, x)$ and $G_{p}(x)=\nabla_{p_{1}} S_{h}(0, p, x)$. Then we have the relation

$$
F_{p}=G_{-p}
$$

Proof. Notice that it is equivalent to prove that $F_{p}=G_{-p}$ or $F_{p}^{-1}=G_{-p}^{-1}$. We prove the second identity. For each $n \geq 1$ we have the decomposition

$$
S_{n}\left(p_{1}, p_{2}, x\right)=S_{n}^{1}\left(p_{1}, p_{2}, x\right)+S_{n}^{2}\left(p_{1}, p_{2}, x\right)+\cdots+S_{n}^{n}\left(p_{1}, p_{2}, x\right),
$$


where $S_{n}^{i}$ is the part of $S_{n}$ which is homogeneous of degree $i$ in the first argument. Now we have that

$$
S_{n}^{i}(-p, p, x)=(-1)^{i} S_{n}^{i}(p, p, x)=0
$$

because of naturality of the generating function. This implies that $S(-p, p, x)=0$. If we differentiate this equation with respect to $p$ we get exactly $F_{p}^{-1}=G_{-p}^{-1}$.

Going back to the check of axioms we get that Axiom 5 is exactly equivalent to $F_{p}=G_{-p}$.

As for Axiom 6, if we pose $i(p, x)=\left(-p, \nabla_{p_{1}} S_{h}(-p, p, s(p, x))\right.$, then

$$
\begin{aligned}
i(p, x)(p, x) & =\left(\nabla_{x} S_{h}(-p, p, s(p, x), s(p, x))\right. \\
& =(0, s(p, x)) \\
& =\epsilon(s(p, x))
\end{aligned}
$$

provided that $x=\nabla_{p_{2}} S_{h}\left(-p, p, \nabla_{p_{2}} S_{h}(p, 0, x)\right)$, which is guaranteed by Lemma 1 .

Similarly for Axiom 7 , if we put $\tilde{i}(p, x)=\left(-p, \nabla_{p_{2}} S(p,-p, t(p, x))\right.$ we get that $(p, x) \tilde{i}(p, x)=\epsilon(t(p, x))$. Now by Lemma 2 we get

$$
\begin{aligned}
& \nabla_{p_{2}} S_{h}(p,-p, t(p, x))=F_{-p} \circ G_{p}(x)=x, \\
& \nabla_{p_{1}} S_{h}(-p, p, s(p, x))=G_{-p} \circ F_{p}(x)=x .
\end{aligned}
$$

Thus $i(p, x)=\tilde{i}(p, x)=(-p, x)$.

Now using the canonical symplectic bracket on $G_{h}$, i.e.,

$$
\{F, G\}_{G_{h}}(p, x)=\left\langle\nabla_{x} F(p, x), \nabla_{p} G(p, x)\right\rangle-\left\langle\nabla_{p} F(p, x), \nabla_{x} G(p, x)\right\rangle,
$$

we can consider the problem of finding a Poisson bracket on $\mathbb{R}^{d}$ such that $s_{h}$ is a Poisson algebra homomorphism, i.e.,

$$
s_{h}^{*}\{f, g\}_{\mathbb{R}^{d}}(p, x)=\left\{s_{h}^{*} f, s_{h}^{*} g\right\}_{G_{h}}(p, x) .
$$

The following proposition answers this question.

Proposition 2. There is a unique Poisson structure on $\mathbb{R}^{d}$ such that $s^{*}$ is a (formal) Poisson map. Moreover this Poisson structure is given by

$$
\{f, g\}_{\mathbb{R}^{d}}(x)=\left\{s_{h}^{*} f, s_{h}^{*} g\right\}_{G_{h}}(0, x)=2 h S_{1}(d f, d g, x) .
$$

Proof. Suppose there exists a Poisson structure $\{,\}_{\mathbb{R}^{d}}$ such that $s^{*}$ is Poisson. This means that $\{f, g\}_{\mathbb{R}^{d}}\left(s_{h}(p, x)\right)=\left\{s_{h}^{*} f, s_{h}^{*} g\right\}_{G_{h}}(p, x)$. In particular if we put $p=0$ we get exactly that $\{f, g\}_{\mathbb{R}^{d}}(x)=\left\{s_{h}^{*} f, s_{h}^{*} g\right\}_{G_{h}}(0, x)=2 h S_{1}(d f, d g, x)$ which shows uniqueness. Now it remains to prove that $\{f, g\}_{\mathbb{R}^{d}}\left(s_{h}(p, x)\right)=\left\{s_{h}^{*} f, s_{h}^{*} g\right\}_{G_{h}}(p, x)$ which proves as well that the induced bracket is Poisson.

Then we have to check that

$$
\left\{s_{h}^{*} f, s_{h}^{*} g\right\}_{G_{h}}\left(0, s_{h}(p, x)\right)=\left\{s_{h}^{*} f, s_{h}^{*} g\right\}_{G_{h}}(p, x)
$$


An easy computation gives us that this equation is equivalent to the following:

$$
\begin{aligned}
& \frac{\partial s_{h}^{l}}{\partial p^{k}}\left(0, s_{h}(p, x)\right)-\frac{\partial s_{h}^{k}}{\partial p^{l}}\left(0, s_{h}(p, x)\right) \\
& \quad=\sum_{i=1}^{d}\left\{\frac{\partial s_{h}^{k}}{\partial x^{i}}(p, x) \frac{\partial s_{h}^{l}}{\partial p^{i}}(p, x)-\frac{\partial s_{h}^{l}}{\partial x^{i}}(p, x) \frac{\partial s_{h}^{k}}{\partial p^{i}}(p, x)\right\} .
\end{aligned}
$$

Differentiating the SGA equation first with respect to $p_{3}$ and then to $p_{2}$ and then putting $p_{1}=p, p_{2}=p_{3}=0$, we get

$$
\begin{aligned}
\nabla_{p_{k}^{1}} \nabla_{p_{l}^{2}} S\left(0,0, s_{h}(p, x)\right)= & \sum_{i=1}^{d} \nabla_{x^{i}} \nabla_{p_{k}^{2}} S(p, 0, x) \nabla_{p_{i}^{1}} \nabla_{p_{l}^{2}} S(p, 0, x) \\
& -\nabla_{p_{k}^{2}} \nabla_{p_{l}^{2}} S(p, 0, x) .
\end{aligned}
$$

Taking the difference between this equation and the same but with the indices $k$ and $l$ interchanged we finish the proof.

\section{Basic Examples}

Let us see in some examples what are the generating functions and the formal symplectic groupoids. We already know what happens in the case of the trivial Poisson structure over $\mathbb{R}^{d}$. The generating function is $S_{0}\left(p_{1}, p_{2}, x\right)=\left(p_{1}+p_{2}\right) x$ and the associated symplectic groupoid is the cotangent bundle $T^{*} \mathbb{R}^{d}$ with structure maps $s(p, x)=x, t(p, x)=x$, $\epsilon(x)=(0, x) i(p, x)=(-p, x)$. The composition is the fiberwise addition.

3.0.1. Constant Poisson structure. Suppose one has a constant Poisson structure $\alpha(x)=\alpha$. The main theorem tells us that the generating function is $S_{h}\left(p_{1}, p_{2}, x\right)=$ $S_{0}\left(p_{1}, p_{2}, x\right)+h p_{1}^{t} \alpha p_{2}$. The multiplication space can then be described as

$$
G_{h}^{(m)}=\left\{\left(\left(p_{1}, x+h \alpha p_{2}\right),\left(p_{2}, x-h \alpha p_{1}\right),\left(p_{1}+p_{2}, x\right)\right),\left(p_{1}, p_{2}, x\right) \in B_{2}\right\} .
$$

By Proposition 1 the structure maps are given by

$$
\begin{aligned}
\epsilon_{h}(x) & =(0, x), \\
i_{h}(p, x) & =(-p, x), \\
s_{h}(p, x) & =x-h \alpha p, \\
t_{h}(p, x) & =x+h \alpha p .
\end{aligned}
$$

3.0.2. Linear Poisson structure. Suppose that we have a linear Poisson structure $\alpha^{i j}(x)=$ $\alpha_{k}^{i j} x^{k}$ on $\mathbb{R}^{d}$ which can then be considered as the dual of a Lie algebra $\mathbb{R}^{d}=\mathcal{G}^{*}$, the bracket on $\mathcal{G}$ being given by $\left[\epsilon^{i}, \epsilon^{j}\right]=2 \alpha_{k}^{i j} \epsilon^{k}$, where $\epsilon^{l}, l=1, \ldots, d$ is a basis of $\mathcal{G}\left(=\mathbb{R}^{* d}\right)$. For this Lie algebra we have the $\mathrm{CBH}$ formula $\exp \left(p_{1}\right) \exp \left(p_{2}\right)=$ $\exp \left(C B H\left(p_{1}, p_{2}\right)\right)$,

$$
C B H\left(p_{1}, p_{2}\right)=p_{1}+p_{2}+\frac{1}{2}\left[p_{1}, p_{2}\right]+\frac{1}{12}\left(\left[p_{1},\left[p_{2}, p_{2}\right]\right]+\left[p_{2},\left[p_{2}, p_{1}\right]\right]\right)+\ldots
$$


It is easy to check directly that

$$
S_{h}\left(p_{1}, p_{2}, x\right)=\left\langle\frac{1}{h} C B H\left(h p_{1}, h p_{2}\right), x\right\rangle,
$$

where $\langle.,$.$\rangle is the usual pairing between \mathcal{G}$ and $\mathcal{G}^{*}$, satisfies the SGA equation. It is equivalent to the associativity of $\mathrm{CBH}$, i.e.,

$$
C B H\left(h p_{1}, C B H\left(h p_{2}, h p_{3}\right)\right)=C B H\left(C B H\left(h p_{1}, h p_{2}\right), h p_{3}\right) .
$$

By the uniqueness of the generating function given by the main theorem we recover a result of V. Kathotia (see [12]):

Proposition 3. For the Poissson structure coming from the dual of a Lie algebra we have

$$
\left\langle\frac{1}{h} C B H\left(h p_{1}, h p_{2}\right)-\left(p_{1}+p_{2}\right), x\right\rangle=\sum_{n \geq 1} \frac{h^{n}}{n !} \sum_{\Gamma \in T_{n, 2}} W_{\Gamma} \hat{B}_{\Gamma}\left(p_{1}, p_{2}, x\right) .
$$

This result is one of the main ingredients to prove that $\mathrm{CBH}$-quantization is a deformation quantization in the case of the dual of a Lie algebra. It allows us to consider the generating function as a generalization of the $\mathrm{CBH}$ formula to the non-linear case.

By Proposition 1 we have that the deformed source and target maps are

$$
\begin{aligned}
& t_{h}(p, x)=\left\langle\frac{1}{h} \nabla_{p_{1}} C B H(0, h p), x\right\rangle=x+h \alpha_{k}^{i j} x^{k} p_{j}+\frac{h^{2}}{3} \alpha_{l}^{u v} \alpha_{v}^{n i} x^{l} p_{u} p_{n}+\ldots, \\
& s_{h}(p, x)=\left\langle\frac{1}{h} \nabla_{p_{2}} C B H(h p, 0), x\right\rangle=x-h \alpha_{k}^{i j} x^{k} p_{j}+\frac{h^{2}}{3} \alpha_{l}^{u v} \alpha_{v}^{n i} x^{l} p_{u} p_{n}+\ldots
\end{aligned}
$$

\section{Perturbative Form of the SGA Equation}

The goal of this section is to formulate a perturbative version of the SGA equation. It is divided in two parts. First we introduce some tools and state the perturbative version of the SGA equation in Proposition 4. The proof is then split into several lemmas.

4.1. Perturbative SGA and Cayley trees. Let us recall that $B_{n}:=\left(\mathbb{R}^{* d}\right)^{n} \times \mathbb{R}^{d}$. First suppose that we are looking for a generating function of the form $S_{h}=S_{0}+$ $h S$ where $S_{0}\left(p_{1}, p_{2}, x\right)=\left(p_{1}+p_{2}\right) x$ is the trivial generating function and $S \in$ $C^{\infty}\left(B_{2}\right)[[h]]$ is a formal series $S=S_{1}+h S_{2}+\ldots$ Inserting $S_{h}$ in the SGA equation we get a new version of this equation for $S, M^{1}(S)=M^{2}(S)$, where $M^{i}: C^{\infty}\left(B_{2}\right)[[h]] \rightarrow$ $C^{\infty}\left(B_{3}\right)[[h]]$ are defined by

$$
\begin{aligned}
M^{1}(S)\left(p_{1}, p_{2}, p_{3}, x ; h\right)= & h S\left(p_{1}, p_{2}, \bar{x}\right)+h S\left(\bar{p}, p_{3}, x\right) \\
& -h^{2} \nabla_{x} S\left(p_{1}, p_{2}, \bar{x}\right) \nabla_{p_{1}} S\left(\bar{p}, p_{3}, x\right), \\
\bar{p}= & p_{1}+p_{2}+h \nabla_{x} S\left(p_{1}, p_{2}, \bar{x}\right), \\
\bar{x}= & x+h \nabla_{p_{1}} S\left(\bar{p}, p_{3}, x\right),
\end{aligned}
$$

and

$$
\begin{aligned}
M^{2}(S)\left(p_{1}, p_{2}, p_{3}, x ; h\right)= & h S\left(p_{2}, p_{3}, \tilde{x}\right)-\tilde{p} \tilde{x}+h S\left(p_{1}, \tilde{p}, x\right) \\
& -h^{2} \nabla_{x} S\left(p_{2}, p_{3}, \tilde{x}\right) \nabla_{p_{2}} S\left(p_{1}, \tilde{p}, x\right), \\
\tilde{p}= & p_{2}+p_{3}+h \nabla_{x} S\left(p_{2}, p_{3}, \tilde{x}\right), \\
\tilde{x}= & x+h \nabla_{p_{2}} S\left(p_{1}, \tilde{p}, x\right) .
\end{aligned}
$$


The idea now is to expand $M^{i}(S)(h), i=1,2$ into powers of $h$ and then to analyze the conditions imposed on $S$ by the equation at each order. For that purpose we are going to introduce some tools and methods that are heavily inspired by the tools used in numerical analysis to determine the order condition of a Runge-Kutta method. The main ingredients are trees which are used to represent the so-called elementary differentials and elementary functions. As these ideas go back to Cayley, we call such trees Cayley trees, in order to distinguish them from Kontsevich trees which will also appear in the story. In the sequel we will mainly follow the notations of [9].***

Definition 3. (1) $A$ graph $t$ is given by a set of vertices $V_{t}=\{1, \ldots, n\}$ and a set of edges $E_{t}$ which is a set of pairs of elements of $V_{t}$. We denote the number of vertices by $|t|$. An isomorphism between two graphs $t$ and $t^{\prime}$ having the same number of vertices is a permutation $\sigma \in S_{|t|}$ such that $\{\sigma(v), \sigma(w)\} \in E_{t^{\prime}}$ if $\{v, w\} \in E_{t}$. Two graphs are called equivalent if there is an isomorphism between them. The symmetries of a graph are the automorphisms of the graph. We denote the group of symmetries by sym $(t)$.

(2) A tree is a graph which has no cycles. Isomorphisms and symmetries are defined the same way as for graphs.

(3) A rooted tree is a tree with one distinguished vertex. An isomorphism of rooted trees is an isomorphism of graphs which sends the root to the root. Symmetries and equivalence are defined correspondingly.

(4) A bipartite graph is a graph $t$ together with a map $\omega: V_{t} \rightarrow\{0, \bullet\}$ such that $\omega(v) \neq \omega(w)$ if $\{v, w\} \in E_{t}$. An isomorphism of bipartite trees is an isomorphism of graphs which respects the coloring, i.e., $\omega(\sigma(v))=\omega(v)$.

The following table summarizes some notations we will use in the sequel.

\begin{tabular}{ll}
\hline$T$ & the set of bipartite trees \\
$R T$ & the set of rooted bipartite trees \\
$R T$ & the set of elements of $R T$ with white root \\
$R T$. & the set of elements of $R T$ with black root \\
\hline
\end{tabular}

$[A]$ : the set of equivalence classes of graphs in $A$ (ex: $[R T])$. They are called topological " $A$ " trees.

The elements of $[R T]$ can be described recursively as follows

(1) $\circ, \bullet \in[R T]$.

(2) If $t_{1}, \ldots, t_{m} \in\left[R T_{\circ}\right]$, then the tree $\left[t_{1}, \ldots, t_{m}\right]_{\bullet} \in[R T]$ where $\left[t_{1}, \ldots, t_{m}\right]_{\bullet}$ is defined by connecting the roots of $t_{1}, \ldots, t_{m}$ with $\bullet$ and saying that $\bullet$ is the new root, and the same if we interchange $\circ$ and $\bullet$.

Now with the help of this recursive description of topological rooted trees we define elementary differentials and elementary generating functions.

Definition 4 (Elementary Differentials (ED)). Let $i=1,2, t \in[R T]$. The elementary differential $D^{i} S(t)$ of $S \in C^{\infty}\left(B_{2}\right)[[h]]$ is defined recursively as follows:

(1) $D^{i} S(\circ)=\nabla_{x} S, D^{i} S(\bullet)=\nabla_{p^{i}} S$,

(2) $D^{i} S(t)=\nabla_{p^{i}}^{(m+1)} S\left(D^{i} S\left(t_{1}\right), \ldots, D^{i} S\left(t_{m}\right)\right)$ if $t=\left[t_{1}, \ldots, t_{m}\right]_{\bullet}$,

(3) $D^{i} S(t)=\nabla_{x}^{(m+1)} S\left(D^{i} S\left(t_{1}\right), \ldots, D^{i} S\left(t_{m}\right)\right)$ if $t=\left[t_{1}, \ldots, t_{m}\right]_{\circ}$, 
where $\nabla_{x}^{(k)} S$ stands for the $k^{\text {th }}$ derivative of $S$ w.r.t. $x$ evaluated at $\left(p_{1}, p_{2}, x\right)$ if $i=1$ and at $\left(p_{2}, p_{3}, x\right)$ if $i=2 . \nabla_{p^{i}}^{(k)} S$ stands for the $k^{\text {th }}$ derivative of $S$ w.r.t. $p^{i}$ evaluated at $\left(p_{1}+p_{2}, p_{3}, x\right)$ if $i=1$ and at $\left(p_{1}, p_{2}+p_{3}\right)$ if $i=2$.

Definition 5 (Elementary Generating Functions (EGF)). Let $i=1,2, t \in[R T]$. The elementary generating function $S^{i}(t)$ of $S \in C^{\infty}\left(B_{2}\right)[[h]]$ is defined recursively as follows:

(1) $S^{1}(\circ)=S\left(p_{1}, p_{2}, x\right), \quad S^{1}(\bullet)=S\left(p_{1}+p_{2}, p_{3}, x\right)$,

(2) $S^{2}(\circ)=S\left(p_{2}, p_{3}, x\right), \quad S^{2}(\bullet)=S\left(p_{1}, p_{2}+p_{3}, x\right)$,

(3) $S^{i}(t)=\nabla_{p^{i}}^{(m)} S\left(D^{i} S\left(t_{1}\right), \ldots, D^{i} S\left(t_{m}\right)\right)$ if $t=\left[t_{1}, \ldots, t_{m}\right]_{\bullet}$,

(4) $S^{i}(t)=\nabla_{x}^{(m)} S\left(D^{i} S\left(t_{1}\right), \ldots, D^{i} S\left(t_{m}\right)\right)$ if $t=\left[t_{1}, \ldots, t_{m}\right]_{\circ}$,

with the same notation as above.

Some examples are given in the following table:

\begin{tabular}{llll}
\hline Diagram & Notation & ED & EGF \\
\hline & {$[\bullet]_{\circ}$} & $\nabla_{x}^{(2)} S \nabla_{p} S$ & $\nabla_{x} S \nabla_{p} S$ \\
\hline & {$[\circ, \circ] \bullet$} & $\nabla_{p}^{(3)} S\left(\nabla_{x} S, \nabla_{x} S\right)$ & $\nabla_{p}^{(2)} S\left(\nabla_{x} S, \nabla_{x} S\right)$ \\
\hline & {$\left[\bullet,[\circ]_{\bullet}\right]_{\circ}$} & $\nabla_{x}^{(3)} S\left(\nabla_{p} S, \nabla_{p}^{(2)} S \nabla_{x} S\right)$ & $\nabla_{x}^{(2)} S\left(\nabla_{p} S, \nabla_{p}^{(2)} S \nabla_{x} S\right)$ \\
\hline
\end{tabular}

Remark that for EGF it is not important which vertex is the root. This is not the case for $\mathrm{ED}$. Let us be more precise.

Definition 6 (Butcher product). Let $u=\left[u_{1}, \ldots, u_{k}\right], v=\left[v_{1}, \ldots, v_{l}\right] \in[R T]$. We denote by

$$
\begin{aligned}
& u \circ v=\left[u_{1}, \ldots, u_{k}, v\right], \\
& v \circ u=\left[v_{1}, \ldots, v_{l}, u\right],
\end{aligned}
$$

the Butcher product. We have not written the obvious conditions on the $u_{i}$ and $v_{i}$ so that the product remains bipartite.

Definition 7 (Equivalence relation on rooted topological trees). We consider the minimal equivalence relation on $[R T]$ such that $u \circ v \sim v \circ u$. 
Properties of this relation. It is clear that

(1) two topological rooted trees are equivalent if it is possible to pass from one to the other by changing the root. More precisely: $t, t^{\prime} \in[R T], t \sim t^{\prime}$ iff there exists a representative $(E, V, r)$ of $t$ and a representative $\left(E^{\prime}, V^{\prime}, r^{\prime}\right)$ of $t^{\prime}$ and a vertex $r^{\prime \prime} \in V$ such that $\left(E, V, r^{\prime \prime}\right)$ and $\left(E^{\prime}, V^{\prime}, r^{\prime}\right)$ are isomorphic rooted trees.

(2) the quotient of $[R T]$ by this equivalence relation is exactly $[T]$.

(3) it follows immediately from the definition $S^{i}(t)=S^{i}\left(t^{\prime}\right)$ if $t \sim t^{\prime}$ for $i=1,2$.

Then, it makes sense to define the EGF on bipartite trees.

Definition 8. Let $S \in C^{\infty}\left(B_{2}\right)[[h]]$ and $t=\left(V_{t}, E_{t}\right) \in T$. Then

$$
S^{1}(t):=\sum_{\beta: E_{t} \rightarrow\{1, \ldots, d\}} \prod_{v \in V_{t}}\left[\left(\prod_{\substack{e \in E_{t} \\ e=\{*, v\}}} D_{\beta(e)}^{1, \omega(v)}\right) S\right],
$$

where

$$
\begin{aligned}
& D_{\beta\left(e_{1}\right)}^{1, \bullet} \ldots D_{\beta\left(e_{k}\right)}^{1, \bullet} S:=\frac{\partial^{k} S}{\partial p_{\beta\left(e_{1}\right)}^{1} \ldots \partial p_{\beta\left(e_{k}\right)}^{1}}\left(p_{1}+p_{2}, p_{3}, x\right), \\
& D_{\beta\left(e_{1}\right)}^{1, \circ} \ldots D_{\beta\left(e_{k}\right)}^{1, \circ} S:=\frac{\partial^{k} S}{\partial x^{\beta\left(e_{1}\right)} \ldots \partial x^{\beta\left(e_{k}\right)}}\left(p_{1}, p_{2}, x\right),
\end{aligned}
$$

and correspondingly for $S^{2}(t)$.

It is clear that this new definition of $S^{i}(t)$ is equivalent to the previously introduced recursive one. This definition is however better if we want to deal with the fact that $S$ is a formal series. Namely we immediately get the relation

$$
\begin{aligned}
h^{|t|} S^{i}(t) & =h^{|t|} \sum_{\beta: E_{t} \rightarrow\{1, \ldots, d\}} \prod_{v \in V_{t}}\left[\left(\prod_{\substack{e \in E_{t} \\
e=\{*, v\}}} D_{\beta(e)}^{i, \omega(v)}\right)\left\{\frac{1}{h} \sum_{n=1}^{\infty} h^{n} S_{n}\right\}\right] \\
& =\sum_{n=|t|}^{\infty} h^{n} \sum_{\substack{n_{1}+\cdots+n_{|t|}=n \\
n_{i} \geq 1}} C_{t}^{i}\left(S_{n_{1}}, \ldots, S_{n_{|t|}}\right),
\end{aligned}
$$

which defines the $C_{t}^{i}$ which are multi-differential maps from $C^{\infty}\left(B_{2}\right)^{|t|}$ to $C^{\infty}\left(B_{3}\right)$.

We can now state the main proposition of this section.

Proposition 4 (Perturbative version of the SGA equation). The formal series $S_{h}=S_{0}+$ $\sum_{n \geq 1} h^{n} S_{n}$ satisfies the SGA equation iff for each $n>0$ we have

$$
\sum_{\substack{t \in T \\|t| \leq n}} \frac{1}{|t| !} \sum_{\substack{n_{1}+\cdots+n_{|t|}=n \\ n_{i} \geq 1}} C_{t}^{1}\left(S_{n_{1}}, \ldots, S_{n_{|t|}}\right)-C_{t}^{2}\left(S_{n_{1}}, \ldots, S_{n_{|t|}}\right)=0 .
$$

Let us remark that for all $f \in C^{\infty}\left(B_{2}\right)$ we have that

$$
C_{\bullet}^{1}(f)+C_{\circ}^{1}(f)-C_{\bullet}^{2}(f)-C_{\circ}^{2}(f)=d S,
$$


where $d: C^{\infty}\left(B_{n}\right) \rightarrow C^{\infty}\left(B_{n+1}\right)$ is a differential (i.e., $\left.d^{2}=0\right)$ defined by the formula

$$
\begin{aligned}
d f\left(p_{1}, \ldots, p_{n+1}\right)= & f\left(p_{2}, \ldots, p_{n+1}\right)-\sum_{i=1}^{n}(-1)^{i+1} f\left(p_{1}, \ldots, p_{i}+p_{i+1}\right) \\
& +(-1)^{n+1} f\left(p_{1}, \ldots, p_{n}\right) .
\end{aligned}
$$

This differential can be interpreted either as the Hochschild differential on symbols of multi-differential operators on $C^{\infty}\left(\mathbb{R}^{d}\right)$ or as the differential of the trivial symplectic groupoid cohomology over $\mathbb{R}^{d}$. This remark allows us to put the previous recursive equations into the form

$$
d S_{n}+H_{n}\left(S_{n-1}, \ldots, S_{1}\right)=0,
$$

which is exactly the analog of the recursive equation involved when considering starproducts.

The remainder of this section is devoted to proving Proposition 4.

4.2. Proof of the Proposition. It follows from a series of little lemmas. We are first interested in expanding

$$
\begin{aligned}
& \bar{p}=p_{1}+p_{2}+h \nabla_{x} S\left(p_{1}, p_{2}, \bar{x}\right), \\
& \bar{x}=x+h \nabla_{p_{1}} S\left(\bar{p}, p_{3}, x\right),
\end{aligned}
$$

and

$$
\begin{aligned}
& \tilde{p}=p_{2}+p_{3}+h \nabla_{x} S\left(p_{2}, p_{3}, \tilde{x}\right), \\
& \tilde{x}=x+h \nabla_{p_{2}} S\left(p_{1}, \tilde{p}, x\right)
\end{aligned}
$$

as a power series in $h$.

The method used is essentially the same as in numerical analysis when one wants to express the Taylor series of the numerical flow of a Runge-Kutta method. Namely the equations above have a form very close to the partitioned implicit Euler method (see [9]).

Definition 9. Let $t=\left[t_{1}, \ldots, t_{m}\right] \in[R T]$. Consider the list $\tilde{t}_{1}, \ldots, \tilde{t}_{k}$ of all nonisomorphic trees appearing in $t_{1}, \ldots, t_{m}$. Define $\mu_{i}$ as the number of times the tree $\tilde{t}_{i}$ appears in $t_{1}, \ldots, t_{m}$. Then we introduce the symmetry coefficient $\sigma(t)$ of $t$ by the following recursive definition:

$$
\sigma(t)=\mu_{1} ! \mu_{2} ! \ldots \sigma\left(\tilde{t}_{1}\right) \ldots \sigma\left(\tilde{t}_{k}\right) .
$$

Moreover $\sigma(\circ)=\sigma(\bullet)=1$.

It is clear that $\sigma(t)$ is the number of symmetries for each representative of $t$ (i.e., $\sigma(t)=\left|\operatorname{Sym}\left(t^{\prime}\right)\right|$ for all $\left.t^{\prime} \in t\right)$.

Lemma 3. There exist unique formal series for $\bar{x}, \bar{p}$ (resp. $\tilde{x}, \tilde{p})$ which satisfy Eqs. (1) and (2) (resp. (2) and (3)). They are given by

$$
\begin{aligned}
& \bar{x}(h)=x+\sum_{t \in\left[R T_{\bullet}\right]} \frac{h^{|t|}}{\sigma(t)} D^{1} S(t), \\
& \bar{p}(h)=p_{1}+p_{2}+\sum_{t \in\left[R T_{\circ}\right]} \frac{h^{|t|}}{\sigma(t)} D^{1} S(t),
\end{aligned}
$$


and by

$$
\begin{aligned}
& \tilde{x}(h)=x+\sum_{t \in\left[R T_{\bullet}\right]} \frac{h^{|t|}}{\sigma(t)} D^{2} S(t), \\
& \tilde{p}(h)=p_{2}+p_{3}+\sum_{t \in\left[R T_{\circ}\right]} \frac{h^{|t|}}{\sigma(t)} D^{2} S(t),
\end{aligned}
$$

respectively.

Proof. Uniqueness is trivial. Let us check that we have the right formal series. We only check Eq. (1). The other computation is similar.

$$
\begin{aligned}
\bar{x}(h)= & x+h \nabla_{p_{1}} S\left(\bar{p}, p_{3}, x\right) \\
= & x+h \sum_{m \geq 0} \frac{1}{m !} \nabla_{p}^{(m+1)} S\left(\sum_{t \in\left[R T_{\circ}\right]} \frac{h^{|t|}}{\sigma(t)} D^{1} S(t), \ldots, \sum_{t \in\left[R T_{\circ}\right]} \frac{h^{|t|}}{\sigma(t)} D^{1} S(t)\right) \\
= & x+\sum_{m \geq 0} \sum_{t_{1} \in\left[R T_{\circ}\right]} \ldots \sum_{t_{m} \in\left[R T_{\circ}\right]} \frac{h^{1+\left|t_{1}\right|+\cdots+\left|t_{m}\right|}}{m ! \sigma\left(t_{1}\right) \ldots \sigma\left(t_{m}\right)} \\
& \times \nabla_{p}^{(m+1)} S\left(D^{1} S\left(t_{1}\right), \ldots, D^{1} S\left(t_{m}\right)\right) \\
= & x+\sum_{m \geq 0} \sum_{t_{1}} \ldots \sum_{t_{m}} \frac{h^{|t|}}{m ! \sigma(t)}\left(\mu_{1} ! \mu_{2} ! \ldots\right) D^{1} S(t), \quad \text { with } t=\left[t_{1}, \ldots, t_{m}\right] \bullet \\
= & x+\sum_{t \in\left[R T_{\bullet}\right]} \frac{h^{|t|}}{\sigma(t)} D^{1} S(t) .
\end{aligned}
$$

We now insert these expansions into $M^{1}$ and $M^{2}$.

\section{Lemma 4.}

$$
M^{i}(S)(h)=\sum_{t \in[R T]} \frac{h^{|t|}}{\sigma(t)} S^{i}(t)-\left(\sum_{t \in\left[R T_{\circ}\right]} \frac{h^{|t|}}{\sigma(t)} D^{i} S(t)\right)\left(\sum_{t \in\left[R T_{\bullet}\right]} \frac{h^{|t|}}{\sigma(t)} D^{i} S(t)\right)
$$

for $i=1,2$.

Proof. Let us do the proof for $M^{1}$. First we compute the different terms arising in the formula for $M^{1}$ in terms of trees: 


$$
\begin{aligned}
& h S\left(p_{1}, p_{2}, \bar{x}\right)= h \sum_{m \geq 0} \frac{1}{m !} \nabla_{x}^{(m)} S\left(\sum_{t \in\left[R T_{\bullet}\right]} \frac{h^{|t|}}{\sigma(t)} D^{1} S(t), \ldots,\right. \\
&\left.\ldots, \sum_{t \in\left[R T_{\bullet}\right]} \frac{h^{|t|}}{\sigma(t)} D^{1} S(t)\right) \\
&=\sum_{m \geq 0} \sum_{t_{1} \in\left[R T_{\bullet}\right]} \ldots \sum_{t_{m} \in\left[R T_{\bullet}\right]} \frac{h^{|t|}}{m ! \sigma(t)}\left(\mu_{1} ! \mu_{2} ! \ldots\right) \\
& \times \nabla_{x}^{(m)} S\left(D^{1} S\left(t_{1}\right), \ldots, D^{1} S\left(t_{m}\right)\right), \quad \text { with } t=\left[t_{1}, \ldots, t_{m}\right] \bullet \\
&= \sum_{t \in\left[R T_{\bullet}\right]} \frac{h^{|t|}}{\sigma(t)} S^{1}(t) .
\end{aligned}
$$

By the same sort of computations we also get

$$
\begin{gathered}
h S\left(\bar{p}, p_{3}, x\right)=\sum_{t \in\left[R T_{\bullet}\right]} \frac{h^{|t|}}{\sigma(t)} S^{1}(t), \\
h \nabla_{x} S\left(p_{1}, p_{2}, \bar{x}\right)=\sum_{t \in\left[R T_{\circ}\right]} \frac{h^{|t|}}{\sigma(t)} D^{1} S(t), \\
h \nabla_{p_{1}} S\left(\bar{p}, p_{3}, x\right)=\sum_{t \in\left[R T_{\bullet}\right]} \frac{h^{|t|}}{\sigma(t)} D^{1} S(t) .
\end{gathered}
$$

The $M^{i}$ 's are expressed as sums over topological rooted bipartite trees. We would like now to regroup the terms of the formula in the previous lemma. To do so we express all terms in terms of topological trees (no longer rooted).

Lemma 5. Let $u \in\left[R T_{\circ}\right]$ and $v \in\left[R T_{\bullet}\right]$. Then $D^{i} S(u) D^{i} S(v)=S^{i}(u \circ v)=S^{i}(v \circ u)$.

Proof. Prove it only for $i=1$. Suppose $u=\left[u_{1}, \ldots, u_{m}\right]_{\circ}, v=\left[v_{1}, \ldots, v_{l}\right]_{\bullet}$, then

$$
\begin{aligned}
D^{1} S(u) D^{1} S(v) & =\nabla_{x}^{(m+1)} S\left(D^{1} S\left(u_{1}\right), \ldots, D^{1} S\left(u_{m}\right)\right) . D^{1} S(v) \\
& =\nabla_{x}^{(m+1)} S\left(D^{1} S\left(u_{1}\right), \ldots, D^{1} S\left(u_{m}\right), D^{1} S(v)\right) \\
& =S^{1}(u \circ v) .
\end{aligned}
$$

Lemma 6. Let $t=\left(V_{t}, E_{t}\right) \in T$. For all $v \in V_{t}$ let $t_{v}$ be the bipartite rooted tree $\left(V_{t}, E_{t}, v\right) \in R T$. For $v \in V_{t}$ and $e=\{u, v\} \in E_{t}$ we have

$$
\begin{gathered}
\frac{|\operatorname{sym}(t)|}{\left|\operatorname{sym}\left(t_{v}\right)\right|}=\mid\left\{v^{\prime} \in V_{t} / t_{v^{\prime}} \text { is isomorphic to } t_{v}\right\} \mid, \\
\frac{|\operatorname{sym}(t)|}{\left|\operatorname{sym}\left(t_{u}\right)\right|\left|\operatorname{sym}\left(t_{v}\right)\right|}=\mid\left\{e^{\prime} \in E_{t} / t_{u^{\prime}} \sqcup t_{v^{\prime}} \text { is isomorphic to } t_{u} \sqcup t_{v}\right\} \mid .
\end{gathered}
$$


Proof. Consider the induced action of the symmetry group of the tree on the set of vertices. Notice that two vertices $v$ and $w$ are in the same orbit iff $t_{v}$ is isomorphic to $t_{w}$. Then the number of vertices of $t$ which lead to a rooted tree isomorphic to $t_{v}$ is exactly the cardinality of the orbit of $v$, which is exactly $|\operatorname{sym}(t)|$ divided by the cardinality of the isotropy subgroup which fixes $v$. But the latter is $\left|\operatorname{sym}\left(t_{v}\right)\right|$ by definition. We then get the first statement.

For the second statement we have to consider the induced action on the edges and apply the same type of argument.

Lemma 7. Let $S \in C^{\infty}\left(B_{2}\right)[[h]]$. The $S G A$ equation for $S$ can be expressed in terms of bipartite Cayley trees as

$$
\sum_{t \in T} \frac{h^{|t|}}{|t| !}\left(S^{1}(t)-S^{2}(t)\right)=0 .
$$

Proof. We have for $i=1,2$,

$$
\begin{aligned}
M^{i}(S)= & \sum_{t \in[R T]} \frac{h^{|t|}}{\sigma(t)} S^{i}(t)-\sum_{u \in\left[R T_{\circ}\right]} \sum_{v \in\left[R T_{\bullet}\right]} \frac{h^{|u|+|v|}}{\sigma(u) \sigma(v)} D^{i} S(u) D^{i} S(v) \\
= & \sum_{\bar{t} \in[T]} h^{|\bar{t}|} S^{i}(\bar{t})\left\{\sum_{t \in \bar{t}} \frac{1}{|\operatorname{sym}(t)|}-\sum_{\substack{u \in\left[R T_{\bullet}\right], v \in\left[R T_{\circ}\right] \\
u \circ v \in \bar{t}}} \frac{1}{|\operatorname{sym}(u)||\operatorname{sym}(v)|}\right\} \\
= & \sum_{t \in T} \frac{h^{|t|}}{|t| !} S^{i}(t)\left\{\sum_{v \in V_{t}} \frac{|\operatorname{sym}(t)|}{\left|\operatorname{sym}\left(t_{v}\right)\right|} \frac{1}{k(t, v)}\right. \\
& \left.-\sum_{e=\{u, v\} \in E_{t}} \frac{|\operatorname{sym}(t)|}{\left|\operatorname{sym}\left(t_{u}\right)\right|\left|\operatorname{sym}\left(t_{v}\right)\right|} \frac{1}{l(t, e)}\right\},
\end{aligned}
$$

where $k(t, v)=\mid\left\{v^{\prime} \in V_{t} / t_{v^{\prime}}\right.$ is isomorphic to $\left.t_{v}\right\} \mid$ and $l(t, e)=\left|\begin{array}{ll}e^{\prime} \in E_{t}\end{array}\right|$ $t_{u^{\prime}} \sqcup t_{v^{\prime}}$ is isomorphic to $\left.t_{u} \sqcup t_{v}\right\} \mid$. Using Lemma 6 and the fact that for a tree the difference between the number of vertices and the number of edges is equal to 1 we get the desired result.

Using now the fact that $S$ is a formal series we immediately get Proposition 4 .

\section{Geometry of Kontsevich Trees}

In this section we present a diagrammatical notation introduced by Kontsevich which allows us to write an explicit solution of the SGA equation.

\subsection{Basic Definitions.}

Definition 10. (1) $A$ Kontsevich graph $\Gamma$ of type $(n, m)$ is a directed graph $\Gamma=$ $\left(E_{\Gamma}, V_{\Gamma}\right)$ which has the following properties:

- It possesses two types of vertices $V_{\Gamma}=V_{\Gamma}^{a} \sqcup V_{\Gamma}^{g}$, the aerial vertices $V_{\Gamma}^{a}=$ $\{1, \ldots, n\}$ and the ground vertices $V_{\Gamma}^{g}=\{\overline{1}, \ldots, \bar{m}\}$. 
- Each aerial vertex possesses exactly two ordered edges starting from it. The edge set can be described as $E_{\Gamma}=\left\{\left(k, \gamma^{i}(k)\right), \quad k=1, \ldots, n, \quad i=1,2\right\}$, where $\gamma^{i}: V_{\Gamma}^{a} \rightarrow V_{\Gamma}$. Sometimes one denotes the two edges of a vertex $k$ by $e_{k}^{1}$ and $e_{k}^{2}$.

- For each aerial vertex $v$ we do not allow small loops (i.e., that $\gamma^{i}(v)=v$ ) and double edges (i.e., that $\gamma^{1}(v)=\gamma^{2}(v)$ ).

We denote the set of Kontsevich graphs of type $(n, m)$ by $G_{n, m}$. If $\Gamma \in G_{n, m}$ then we set $|\Gamma|:=n$.

(2) Let $A \in V_{\Gamma}$. We call $\Gamma_{/ A}$ the restriction of $\Gamma$ to $A$. It is the graph with vertex set $A$ and edges $E_{\Gamma} \cap A \times A$. We call $\Gamma_{(A)}$ the contraction of $\Gamma$ to $A$. It is the graph with vertex set $\left(V_{\Gamma} \backslash A\right) \sqcup\{*\}$ (the vertices of $A$ are contracted to a single vertex $*$ ) and edges $(i, j) \in E_{\Gamma}$, where $i$ is replaced by the new vertex $*$ in $\Gamma_{(A)}$ if $i \in A$ and the same for $j$ (simple loops are deleted). Note that the resulting graphs might not be Kontsevich graphs.

(3) We denote by $\Delta(\Gamma)=\left(V_{\Gamma}^{a}, E_{\Gamma}^{a}\right)$ the restriction of $\Gamma \in G_{n, m}$ to the aerial vertices. Sometimes we write $E_{\Gamma}^{g}=E_{\Gamma} \backslash E_{\Gamma}^{a}$. We say that a Kontsevich graph $\Gamma$ is connected if $\Delta(\Gamma)$ is connected in the usual sense. We say that a connected Kontsevich graph $\Gamma$ is a tree if $\Delta(\Gamma)$ is a tree (i.e., a graph without cycle). Denote by $C_{n, m}$ the set of connected Kontsevich graph of type $(n, m)$ and by $T_{n, m}$ the set of Kontsevich trees of type $(n, m)$.

Given a Poisson structure $\alpha$ on $\mathbb{R}^{d}$ one can associate to each graph $\Gamma \in G_{n, m}$ an $m$-multidifferential operator on $C^{\infty}\left(\mathbb{R}^{d}\right)$. The general formula is the following

$$
B_{\Gamma}\left(f_{1} \ldots, f_{m}\right):=\sum_{I: E_{\Gamma} \rightarrow\{1, \ldots, d\}}\left[\prod_{k \in V_{\Gamma}^{a}}\left(\prod_{\substack{e \in E_{\Gamma} \\ e=(*, k)}} \partial_{I(e)}\right) \alpha^{I\left(e_{k}^{1}\right) I\left(e_{k}^{2}\right)}\right] \times \prod_{i \in V_{\Gamma}^{g}}\left(\prod_{\substack{e \in E_{\Gamma} \\ e=(*, i)}} \partial_{I(e)}\right) f_{i} .
$$

We call $\hat{B}_{\Gamma}$ the symbol of $B_{\Gamma}$. It can be defined by the formula

$$
B_{\Gamma}\left(e^{p_{1} x}, \ldots, e^{p_{m} x}\right)=\hat{B}_{\Gamma}\left(p_{1}, \ldots, p_{m}, x\right) e^{\left(p_{1}+\cdots+p_{m}\right) x} .
$$

Example 1. Take the graph

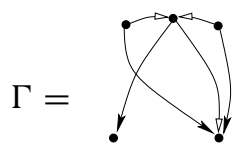

then we have

$$
\hat{B}_{\Gamma}\left(p_{1}, p_{2}, x\right)=\sum_{1 \leq i, j, k, l, m, n \leq d} \alpha^{i j}(x) \partial_{n} \partial_{j} \alpha^{k l}(x) \alpha^{m n}(x) p_{k}^{1} p_{i}^{2} p_{l}^{2} p_{m}^{2} .
$$

Associated to each Kontsevich graph $\Gamma \in G_{n, m}$ there is also a number, the Kontsevich weight $W_{\Gamma}$. In these notes we only need to define these weights for graphs of type $(n, 2)$. The generalization is however straightforward. We do this in several steps.

(1) Take a Kontsevich graph $\Gamma \in G_{n, 2}$ and identify its vertices $1, \ldots, n \in V_{\Gamma}$ with $n$ complex numbers $z_{1}, \ldots, z_{n}$ lying in the upper half complex plane $\mathcal{H}=\{z \in$ $\mathbb{C} / \operatorname{Im}(z)>0\}$ (we require that $z_{i} \neq z_{j}$ if $i \neq j$ ). Identify further $\overline{1}$ and $\overline{2}$ with 0 and 1 in $\mathbb{R}$. 
(2) Consider now the hyperbolic metric on $\mathcal{H}$. The geodesic joining two points $p, q \in$ $\mathcal{H}$ is in this metric either the half circle intersecting orthogonally the real line and passing through $p$ and $q$ or the line orthogonal to the real line passing through $p$ and $q$. We can now associate the oriented edges $e_{k}^{i}=\left(k, \gamma^{i}(k)\right)$ to the oriented geodesics joining $z_{k}$ and $z_{\gamma^{i}(k)}$. We call such an embedding of $\Gamma$ a configuration of $\Gamma$. We can then identify the configuration space of a Kontsevich graph $\Gamma$ with $\mathcal{H}^{n} \backslash D^{n}$, where $\mathcal{H}^{n}$ is $n$ times the Cartesian product of $\mathcal{H}$ and

$$
D^{n}:=\left\{\left(z_{1}, \ldots, z_{n}\right) \in \mathcal{H}^{n} / \exists i, j \quad i \neq j \quad \text { and } \quad z_{i}=z_{j}\right\} .
$$

Notice that $\mathcal{H}^{n} \backslash D^{n}$ is a real non-compact manifold of dimension $2 n$. We can however compactify it into a compact manifold with corners $\overline{\mathcal{H}^{n} \backslash D^{n}}$ such that the open stratum is exactly $\mathcal{H}^{n} \backslash D^{n}$.

(3) For each edge $e_{k}^{i}=\left(k, \gamma^{i}(k)\right)$ we can define an "angle function" on $\mathcal{H}^{n} \backslash D^{n}$ by $\psi_{z_{k}}^{i}\left(z_{1}, \ldots, z_{n}\right):=\phi^{h}\left(z_{k}, z_{\gamma^{i}(k)}\right)$, where $\phi^{h}\left(z_{k}, z_{\gamma^{i}(k)}\right)$ is the oriented hyperbolic angle between the geodesic joining $z_{k}$ and $\infty$ and the geodesic joining $z_{k}$ and $z_{\gamma^{i}(k)}$. So $\phi^{h}(p, q)=\arg \left(\frac{q-p}{q-\bar{p}}\right)$.

(4) We can now consider the 1 -forms $d \psi_{z_{k}}^{i} \in \Omega^{1}\left(\mathcal{H}^{n} \backslash D^{n}\right)$ which can be extended on the compactified space. Then the Kontsevich weight of $\Gamma$ is defined by

$$
W_{\Gamma}:=\frac{1}{(2 \pi)^{2 n}} \int_{\frac{\mathcal{H}^{n} \backslash D^{n}}{i=1}} \bigwedge_{i=1}^{n}\left(d \psi_{z_{k}}^{1} \wedge d \psi_{z_{k}}^{2}\right) .
$$

Further explanations about these operators and weights can be found in [13]. However we still need a lemma which is also proven in (or follows directly from) [13].

Definition 11. Let $\Gamma \in G_{n, 3}$. We denote by $\operatorname{sub}(\Gamma)_{\{\overline{1}, \overline{2}\}}$ the set of the subset $S$ of $V_{\Gamma}^{a}$ such that $\Gamma_{/\{\overline{1}, \overline{2}\} \sqcup S}$ and $\Gamma_{(\{\overline{1}, \overline{2}\} \sqcup S)}$ are still Kontsevich graphs of type $(n, 2)$. We define similarly sub $(\Gamma)_{\{\overline{2}, \overline{3}\}}$.

\section{Lemma 8.}

$$
\sum_{\Gamma \in G_{n, 3}}\left(\sum_{S \in \operatorname{sub}(\Gamma)_{\{\overline{1}, \overline{2}\}}} W_{\Gamma_{/\langle\overline{1}, \overline{2}\} \sqcup S}} W_{\Gamma_{(\{\overline{1}, \overline{2}\} \sqcup S)}}-\sum_{S \in \operatorname{sub}(\Gamma)_{\{\overline{2}, \overline{3}\}}} W_{\Gamma_{/\{\overline{2}, \overline{3}\} \cup S}} W_{\Gamma_{(\overline{1}, \overline{3}\} \cup S)}}\right) \hat{B}_{\Gamma}=0 .
$$

5.2. Factorization into connected components of graphs of type $(n, 2)$. We describe here a procedure which allows us to decompose a graph of type $(n, 2)$ into $l$ graphs $\Gamma_{1}, \ldots, \Gamma_{l}$ of the same type, its connected components in a slightly unusual sense. Take $\Gamma \in G_{n, 2}$. Then

(1) Consider the usual connected components of $\Delta(\Gamma)$. We can number them in a unique way using the following rule: Let $\Delta^{i}(\Gamma), \Delta^{j}(\Gamma)$ be two connected components of $\Delta(\Gamma)$. We impose that $i<j$ iff $\min \left\{V_{\Delta^{i}(\Gamma)}\right\}<\min \left\{V_{\Delta^{j}(\Gamma)}\right\}$.

(2) For each connected component $\Delta^{i}(\Gamma)$ of $\Delta(\Gamma)$ we can reconstruct a Kontsevich graph which we denote by $\Gamma_{i}$ :

(a) To begin with, add to each $\Delta^{i}(\Gamma)$ the vertices and edges that we removed considering $\Delta(\Gamma)$. Let $\hat{\Gamma}_{i}$ be this graph.

(b) Relabel the vertices of $\hat{\Gamma}_{i}$ by $1,2, \ldots,\left|\Delta^{i}(\Gamma)\right|$ preserving the relative order of the vertices of $\Delta^{i}(\Gamma)$. One gets a new Kontsevich graph $\Gamma_{i}$. 
Definition 12. (1) Let $\Gamma \in G_{n, 2}$. We call the $\Gamma_{i}$ 's as constructed above the connected factors of $\Gamma$. Because of the numbering of the $\Delta^{i}(\Gamma)$ the connected factors of a Kontsevich graph $\Gamma$ are uniquely numbered. The connected factors of $\Gamma$ are connected Kontsevich graphs.

(2) We denote by $G_{n, 2}\left(n_{1}, \ldots, n_{k}\right)$ the graphs $\Gamma$ of $G_{n, 2}$ which have $k$ connected factors and such that the $i^{\text {th }}$ connected factor $\Gamma_{i}$ is a Kontsevich graph of order $n_{i}$.

(3) We call the factorization map the map $D$ defined by $D(\Gamma)=\left(\Gamma_{1}, \ldots, \Gamma_{k}\right)$, where the $\Gamma_{i}$ are the connected factors of $\Gamma$.

Similar considerations about connected Kontsevich graphs and connected factorization can be found in [12]. In particular one can find the following lemma:

Lemma 9 (Factorization Lemma). Let $\Gamma \in G_{n, 2}$ and $D(\Gamma)=\left(\Gamma_{1}, \ldots, \Gamma_{k}\right)$ be its connected factorization. Then we have

(1) $\mathcal{W}_{\Gamma}=\mathcal{W}_{\Gamma_{1}} \ldots \mathcal{W}_{\Gamma_{k}}$,

(2) $\hat{B}_{\Gamma}=\hat{B}_{\Gamma_{1}} \ldots \hat{B}_{\Gamma_{k}}$.

5.3. Number of graphs leading to the same connected factorization. We are looking for the number of graphs of $G_{n, 2}$ which lead to the same connected factorization. This number plays a crucial role while proving the existence of the generating function.

It is clear that $D(\Gamma)=D\left(\Gamma^{\prime}\right)$ only if $\Gamma, \Gamma^{\prime} \in G_{n, 2}\left(n_{1}, \ldots, n_{k}\right)$ for some $n_{1}, \ldots, n_{k}$. Therefore the problem of counting the number of Kontsevich graphs of type $(n, 2)$ that lead to the same factorization can be stated in the following terms:

$$
\begin{aligned}
& \text { Given }\left(\Gamma_{1}, \ldots, \Gamma_{k}\right) \in C_{n_{1}, 2} \times \cdots \times C_{n_{k}, 2}, \text { what is the number } \\
& \text { of elements of } D^{-1}\left(\Gamma_{1}, \ldots, \Gamma_{k}\right) \text { ? }
\end{aligned}
$$

The answer is contained in the following remarks:

Notice that the permutation group $S_{n}$ acts on $G_{n, 2}$ by permuting the aerial vertices.

Let $\Gamma \in G_{n, 2}\left(n_{1}, \ldots, n_{k}\right)$. All the graphs $\Gamma^{\prime} \in G_{n, 2}\left(n_{1}, \ldots, n_{k}\right)$ which give the same connected factorization as $\Gamma$ are generated by a subset of $S_{n}$, i.e.,

$$
\forall \Gamma^{\prime} \in G_{n, 2}\left(n_{1}, \ldots, n_{k}\right) \text { s.t. } D(\Gamma)=D\left(\Gamma^{\prime}\right) \quad \exists \sigma \in P \text { s.t. } \sigma \Gamma=\Gamma^{\prime} .
$$

This subset $P \subset S_{n}$ is defined by the constraints:

(1) The permutation must preserve the relative order of the vertices of $V_{\Gamma_{i}}$.

(2) Consider the set of the minimum vertex of each $V_{\Gamma_{i}}$. The permutation must preserve the relative order of this set.

It remains then to count the number of such permutations. The second constraint restricts the number of allowed permutations to $\frac{n !}{k !}$. The first further restricts to $\frac{n !}{k ! n_{1} ! \ldots n_{k} !}$. Thus

$$
\left|D^{-1}\left(\Gamma_{1}, \ldots, \Gamma_{k}\right)\right|=\frac{n !}{k ! n_{1} ! \ldots n_{k} !} .
$$

As this number reappears in another context let us denote it by $d\left(n_{1}, \ldots, n_{k}\right)$ and call it the decomposition coefficient. 
5.4. Contraction-restriction decomposition of trees of type $(n, 3)$. Here begin some new considerations about Kontsevich graphs. We will see that in each Kontsevich tree of type $(n, 3)$ lies, hidden, two Cayley trees which encode the contraction and restriction of the tree leading to Kontsevich trees. These two Cayley trees allow us to make a link between the perturbative SGA equation which is expressed in terms of Cayley trees and the proposed solution expressed in terms of Kontsevich trees. The main results of this section are then summarized in Definition 14 and Proposition 5. But let us begin first to establish a few little facts necessary to make any statement.

Lemma 10. Let $\Gamma \in T_{n, m}$, then

(1) $\left|E_{\Gamma}^{a}\right|=n-1$,

(2) $\left|E_{\Gamma}^{g}\right|=n+1$.

Proof. For the first assertion one notices that $\Delta(\Gamma)$, which has $n$ vertices, is connected, so there are at least $n-1$ edges connecting these vertices. Now, if we add an edge, we create a cycle which contradicts the fact that $\Delta(\Gamma)$ is a tree. The second assertion follows from the identity $\left|E_{\Gamma}^{a}\right|+\left|E_{\Gamma}^{g}\right|=2 n$.

Corollary 1. There is no Kontsevich tree of type $(n, 1)$ (i.e. $\left.T_{n, 1}=\emptyset\right)$.

Proof. As $\left|E_{\Gamma}^{g}\right|=n+1$ and $\left|V_{\Gamma}^{a}\right|=n$, one aerial vertex has its two edges landing at the only ground vertex and we do not allow double edges.

Corollary 2. Suppose $\Gamma \in T_{n, 2}$. Then $E_{\Gamma}^{g}$ has at least one edge landing at $\overline{1}$ and one edge landing at $\overline{2}$.

Proof. Without loss of generality, suppose that all edges of $E_{\Gamma}^{g}$ land at $\overline{1}$ then $\Gamma_{/ V_{\Gamma}^{a} \sqcup \overline{1}} \in$ $T_{n, 1}=\emptyset$.

Corollary 3. Suppose $\Gamma \in T_{n, 2}$. There is at least one $v \in V_{\Gamma}^{a}$ such that $\gamma^{1}(v)=\overline{1}$, $\gamma^{2}(v)=\overline{2}$.

Proof. As $\left|E_{\Gamma}^{g}\right|=n+1$ and $\left|V_{\Gamma}^{a}\right|=n$, there is one aerial vertex where both edges are ground edges. Those two edges can not land at the same ground vertex as we prevent double edges.

Definition 13. (1) Let be $\Gamma \in G_{m, n}$. One defines the following transitive relation among the vertices of $\Gamma: v<w$ iff there exists $a_{1}, \ldots, a_{k} \in V_{\Gamma}$ such that

$$
\left(w, a_{1}\right), \ldots,\left(a_{i}, a_{i+i}\right), \ldots,\left(a_{k}, v\right) \in E_{\Gamma} .
$$

(2) Let be $\Gamma \in G_{n, m}$. Let us denote by

$$
\begin{aligned}
& \operatorname{star}_{i n}(v):=\left\{w \in V_{\Gamma} \quad \text { s.t. } \quad v<w\right\}, \\
& \operatorname{star}_{\text {out }}(v):=\left\{w \in V_{\Gamma} \quad \text { s.t. } \quad w<v\right\} \text {. }
\end{aligned}
$$

Lemma 11. Let $\Gamma \in T_{n, 3}$. Denote $N_{\overline{1}}:=\operatorname{star}_{i n}(\overline{1}), B_{\overline{1}}:=V_{\Gamma}^{a} \backslash N_{\overline{1}}$ and $\Gamma_{B_{\overline{1}}}^{1}, \ldots, \Gamma_{B_{\overline{1}}}^{l}$

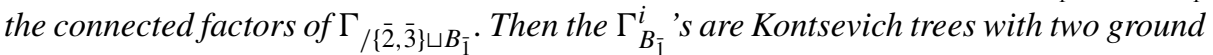
vertices (provided that $B_{\overline{1}} \neq \emptyset$ ). The same statement holds if we replace $B_{\overline{1}}$ by $B_{\overline{3}}$ and make the restriction around $\{\overline{1}, \overline{2}\} \sqcup B_{\overline{3}}$. 
Proof. Take $\Gamma_{B_{\overline{1}}}^{i}$. As there are no edges $(v, w)$ starting from $B_{\overline{1}}$ and landing at $N_{\overline{1}} \sqcup \overline{1}$ (otherwise $v>w>\overline{1} \Rightarrow v \in N_{\overline{1}}$ ), all the vertices of $B_{\overline{1}}$ conserve their two edges when passing to the restriction $\Gamma_{/\{\overline{2}, \overline{3}\} \sqcup B_{\overline{1}}}$. It remains to be shown that all the edges $E_{\Gamma_{B_{\overline{1}}}^{i}}^{g}$ are not landing exclusively at one of $\overline{1}$ or $\overline{2}$. But Corollary 3 prevents this phenomenon from happening.

Trivial little facts. We define for convenience $B_{\overline{1}}^{i}:=V_{\Gamma_{B_{\overline{1}}}^{i}}^{a}, i=1, \ldots, l$ and $N_{\overline{1}}^{j}:=$ $V_{\Gamma_{N_{\overline{1}}}^{j}}^{a}, j=1, \ldots, k$, where $\Gamma_{N_{1}}^{j}$ are the connected factors of $\Gamma_{\left(\{\overline{2}, \overline{3}\} \sqcup B_{\overline{1}}\right)}$. We see that:

(1) There is at most one edge from $\Gamma$ starting from one $N_{\overline{1}}^{j}$ to a $B_{\overline{1}}^{i}$ (otherwise one introduces a cycle).

(2) There is no edge from an $N_{\overline{1}}^{j}$ to another $N_{\overline{1}}^{i}$ (they are connected factors).

(3) There is no edge from a $B_{1}^{j}$ to another $B_{\frac{1}{1}}^{i}$ (they are connected factors).

(4) There is no edge from a $B_{\overline{1}}^{j}$ to a $N_{\overline{1}}^{i}$ (otherwise one vertex of $B_{\overline{1}}^{j}$ should be in $N_{\overline{1}}^{i}$ ).

Corollary 4 (Contraction/Restriction trees). Let $\Gamma \in T_{n, 3}$. We can make the following construction:

- identifying each $N_{\overline{1}}^{j}, j=1, \ldots, k$ and $B_{\overline{1}}^{i}, i=1, \ldots, l$ with respectively black vertex and white vertex,

- putting an edge between black vertex and white vertex iff there is one edge between the corresponding sets $N_{\overline{1}}^{j}$ and $B_{\overline{1}}^{i}$,

- labelling the black and white vertices such that $i<j$ iff the minimum of the set corresponding to $i$ is inferior to the minimum of the set corresponding to $j$,

we get a Cayley tree $t_{\Gamma}^{2} \in T$. This tree $t_{\Gamma}^{2}$ is called the second contraction/restriction tree of $\Gamma$. If we start the construction from $B_{\overline{3}}$ and $N_{\overline{3}}$ we get $t_{\Gamma}^{1}$, the first contraction/restriction tree of $\Gamma$.

Example 2. The following graph $\Gamma$ illustrates these phenomenon:
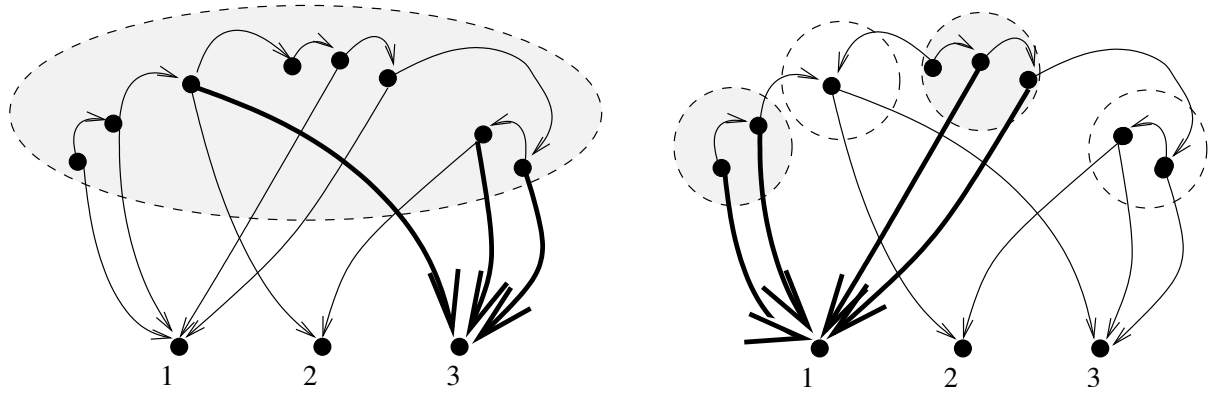

For this graph we have that the two contraction/restriction trees are 


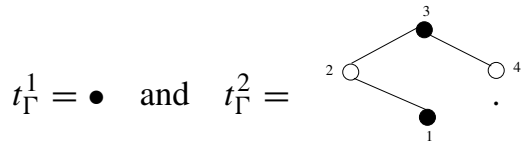

Lemma 12. Let $\Gamma \in T_{n, 3}$. Denote $N_{\overline{1}}:=\operatorname{star}_{i n}(\overline{1}), B_{1}:=V_{\Gamma}^{a} \backslash N_{\overline{1}}$ and $\Gamma_{N_{\overline{1}}}^{1}, \ldots, \Gamma_{N_{\bar{k}}}^{1}$ the connected factor of $\Gamma_{\left(\{\overline{2}, \overline{3}\} \sqcup B_{\overline{1}}\right)}$.

Then the $\Gamma_{N_{\overline{1}}}^{i}$ 's are Kontsevich trees with two ground vertices (provided that $B_{\overline{1}} \neq \emptyset$ ). The same statement holds if we replace $B_{\overline{1}}$ by $B_{\overline{3}}$ and make the contraction around $\{\overline{1}, \overline{2}\} \sqcup B_{\overline{3}}$.

Proof. From the vertices in $N_{\overline{1}}^{i}:=V_{\Gamma_{N_{\overline{1}}}^{i}}$, there is at least one edge landing at $\overline{1}$ and at most one landing at each $B_{\overline{1}}^{\mu}$. The only bad thing that can happen is then there is $v \in N_{\overline{1}}^{i}$ such that $\gamma^{1}(v) \in B_{\overline{1}}^{\mu}$ and $\gamma^{1}(v) \in B_{\overline{1}}^{v}$. But then $v$ has no edge left starting from it, which implies that $\overline{1} \notin \operatorname{star}_{\text {out }}(v)$.

Definition 14. Let $\Gamma \in T_{n, 3}$. We define the contraction/restriction decomposition maps

$$
P^{i}(\Gamma)=\left(t_{\Gamma}^{i}, \Gamma_{1}, \ldots, \Gamma_{m}\right), \quad i=1,2,
$$

where $t_{\Gamma}^{i} \in T$ is the $i^{\text {th }}$ contraction/restriction-tree of $\Gamma$ and the $\Gamma^{j}$ are the connected factor of the contraction and the restriction of $\Gamma$ around $\{\overline{1}, \overline{2}\} \sqcup B_{\overline{3}}$ for $i=1$ and around $\{\overline{2}, \overline{3}\} \sqcup B_{\overline{1}}$ for $i=2$. We index these connected factors with the usual convention, that is $k<l$ if the minimum of the aerial vertices of $\Gamma_{k}$ is less than the minimum of the aerial vertices of $\Gamma_{l}$.

We denote by $T_{n, 3}^{i}\left(t, \Gamma_{1}, \ldots, \Gamma_{|t|}\right)$ the subset of $T_{n, 3}$ such that $P^{i}(\Gamma)=\left(t, \Gamma_{1}, \ldots\right.$, $\left.\Gamma_{|t|}\right)$ for $i=1,2$.

Example 3. For the previous graph $\Gamma$ we get

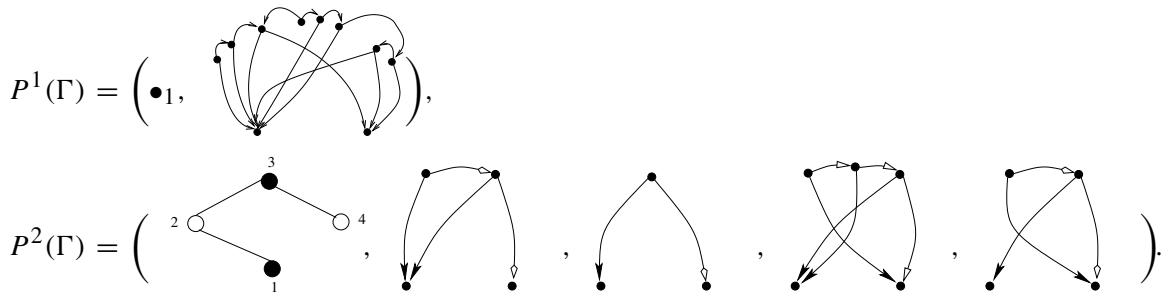

Proposition 5. Let $\Gamma \in T_{n, 3}$. Then in the notation used above we have

(1) Let $\Gamma \in T_{n, 3}^{1}\left(t ; \Gamma_{1}, \ldots, \Gamma_{|t|}\right)$ then

$$
W_{\Gamma_{1}} \ldots W_{\Gamma_{|t|}}=W_{\Gamma / B_{\overline{3}} \cap\{\overline{1}, \overline{2}\}} W_{\Gamma(B \overline{3} \cap\{\overline{1}, \overline{2}\})} .
$$

Let $\Gamma \in T_{n, 3}^{2}\left(t ; \Gamma_{1}, \ldots, \Gamma_{|t|}\right)$ then

$$
W_{\Gamma_{1}} \ldots W_{\Gamma_{|t|}}=W_{\Gamma / B_{\overline{1}} \cap\{\overline{2}, \overline{3}\}} W_{\Gamma\left(B_{\overline{1}} \cap\{\overline{2}, \overline{3}\}\right)} \cdot
$$

(2) We have the following equations for the Kontsevich weights

$$
\sum_{\Gamma \in T_{n, 3}}\left(W_{\Gamma_{\left(\{\overline{1}, \overline{2}\} \sqcup B_{\overline{3}}\right)}} W_{\Gamma_{/\{\overline{1}, \overline{2}\} \sqcup B_{\overline{3}}}}-W_{\Gamma_{\left(\{\overline{2}, \overline{3}\} \cup B_{\overline{1}}\right)}} W_{\Gamma_{/[\overline{2}, \overline{3}\} \cup B_{\overline{1}}}}\right) \hat{B}_{\Gamma}=0 .
$$


(3) The following relates Cayley trees and Kontsevich trees, for all $t \in T$ we have

$$
C_{t}^{i}\left(\hat{B}_{\Gamma_{1}}, \ldots, \hat{B}_{\Gamma_{|t|}}\right)=d\left(n_{1}, \ldots, n_{|t|}\right) \sum_{\Gamma \in T_{n, 3}^{i}\left(t ; \Gamma_{1}, \ldots, \Gamma_{|t|}\right)} \hat{B}_{\Gamma} .
$$

Proof. (1) is trivial.

(2) is a consequence of Lemma 8 once one has proved that $\operatorname{sub}(\Gamma)_{\{\overline{1}, \overline{2}\}}=\left\{B_{\overline{3}}\right\}$ and $\operatorname{sub}(\Gamma)_{\{\overline{2}, \overline{3}\}}=\left\{B_{\overline{1}}\right\}$. By Lemmas 11 and 12 one has already that $B_{\overline{1}} \in \operatorname{sub}(\Gamma)_{\{\overline{2}, \overline{3}\}}$ and $B_{\overline{3}} \in \operatorname{sub}(\Gamma)_{\{\overline{2}, \overline{3}\}}$. It remains to check that they are the only ones. Let us prove that only for $B_{\overline{1}}$.

Suppose there is another subset $K \subset V_{\Gamma}^{a}$ such that $\Gamma_{(\{\overline{2}, \overline{3}\} \sqcup K)}$ and $\Gamma_{/\{\overline{2}, \overline{3}\} \sqcup K}$ are Kontsevich trees. This implies that in the process of

(a) restriction around $\{\overline{2}, \overline{3}\} \sqcup K$, one should not lose an edge

(b) contraction around $\{\overline{2}, \overline{3}\} \sqcup K$, one should not end up with a double edge.

(A) Suppose that $K \cap N_{\overline{1}} \neq \emptyset$. Take $v \in K \cap N_{\overline{1}}$ then $\operatorname{star}_{\text {out }}(v)$ is a subset of $K$ otherwise we lose an edge when doing the restriction around $\{\overline{2}, \overline{3}\} \sqcup K$. But $\overline{1} \in \operatorname{star}_{\text {out }}(v)$ which implies that $\overline{1} \in K$ otherwise we lose an edge when doing the restriction. Contradiction with $K \subset V_{\Gamma}^{a}$.

(B) By (A) we have that $K \subset B_{\overline{1}}$. Suppose that $K$ is strictly contained in $B_{\overline{1}}$. Then $\left(\Gamma_{\left./\{\overline{2}, \overline{3}\} \sqcup B_{\overline{1}}\right)}\right)_{(K \sqcup\{\overline{2}, \overline{3}\})}$ is a subgraph of $\Gamma_{(K \sqcup\{\overline{2}, \overline{3}\})}$. But as there are no edges starting

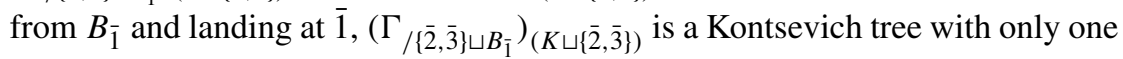
ground vertex which implies that it is not a Kontsevich tree. Contradiction.

(3) First remark that $\sum_{\Gamma \in T_{n, 3}^{i}\left(t, \Gamma_{1}, \ldots, \Gamma_{|t|}\right)} B_{\Gamma}=d\left(n_{1}, \ldots, n_{|t|}\right) \sum_{\Gamma \in A} B_{\Gamma}$, where $A$ is the subset of trees $\Gamma \in T_{n, 3}^{i}\left(t, \Gamma_{1}, \ldots, \Gamma_{|t|}\right)$ such that all vertices in $V_{\Gamma}$ corresponding to these of $V_{\Gamma_{i}}$ are less than these corresponding to $V_{\Gamma_{j}}$ if $i<j$. It is clear that letting all the permutations of $S_{n}$ which preserve the relative order of the minimal vertex of each $V_{\Gamma_{i}}$ and the relative order of the vertices in $V_{\Gamma_{i}}$ act, we get all trees of $T_{n, 3}^{i}\left(t, \Gamma_{1}, \ldots, \Gamma_{|t|}\right)$. We have already counted the number of such permutations; it is exactly the decomposition coefficient $d\left(n_{1}, \ldots, n_{|t|}\right)$.

The identity $\sum_{\Gamma \in A} B_{\Gamma}=C_{t}^{i}\left(\hat{B}_{\Gamma_{1}}, \ldots, \hat{B}_{\Gamma_{k}}\right)$ follows from the Leibniz rule.

\section{Proof of Theorem 1}

Let us restate the main theorem.

Theorem 1. Given a Poisson structure $\alpha$ on $\mathbb{R}^{d}$ there exists a unique natural deformation of the trivial generating function such that the first order is precisely $\alpha$. Moreover we have an explicit formula for this deformation

$$
S_{h}\left(p_{1}, p_{2}, x\right)=x\left(p_{1}+p_{2}\right)+\sum_{n=1}^{\infty} \frac{h^{n}}{n !} \sum_{\Gamma \in T_{n, 2}} W_{\Gamma} \hat{B}_{\Gamma}\left(p_{1}, p_{2}, x\right)
$$

where $T_{n, 2}$ is the set of Kontsevich trees of type $(n, 2), W_{\Gamma}$ is the Kontsevich weight of $\Gamma$, and $\hat{B}_{\Gamma}$ is the symbol of the bidifferential operator $B_{\Gamma}$ associated to $\Gamma$. 
Proof. Existence of the solution. Let us verify that the proposed solution satisfies the perturbative version of the SGA equation. Denote

$$
M_{n}^{i}(S)=\sum_{\substack{t \in T \\|t| \leq n}} \frac{1}{|t| !} \sum_{\substack{n_{1}+\cdots+n_{|t|}=n \\ n_{i} \geq 1}} C_{t}^{i}\left(S_{n_{1}}, \ldots, S_{n_{|t|}}\right) .
$$

Let us compute $M_{n}^{1}(S)$ for the proposed solution

$$
\begin{aligned}
& M^{1}(S)_{n}=\sum_{\substack{t \in T \\
|t| \leq n}} \frac{1}{|t| !} \sum_{\substack{n_{1}+\cdots+n_{|t|}=n \\
n_{i} \geq 1}} \sum_{\substack{\Gamma_{i} \in T_{n_{i}, 2}, 2 \\
i=1, \ldots,|t|}} \frac{W_{\Gamma_{1}} \ldots W_{\Gamma_{|t|} \mid}}{n_{1} ! \ldots n_{|t|} !} C_{t}^{1}\left(\hat{B}_{\Gamma_{1}}, \ldots, \hat{B}_{\Gamma_{|t|}}\right) \\
& =\sum_{\substack{t \in T \\
|t| \leq n}} \sum_{\substack{n_{1}+\cdots+n_{|t|}=n \\
n_{i} \geq 1}} \sum_{\substack{\Gamma_{i} \in T_{n_{i}, 2} \\
i=1, \ldots,|t|}} \frac{W_{\Gamma_{1}} \ldots W_{\Gamma_{|t|}}}{\left(n_{1}+\cdots+n_{|t|}\right) !} \sum_{\substack{\Gamma \in T_{n, 3}^{1}\left(t ; \Gamma_{1}, \ldots, \Gamma_{|t|}\right) \\
n_{\Gamma}}} B_{\Gamma} \\
& =\sum_{\substack{t \in T \\
|t| \leq n}} \frac{1}{n !} \sum_{\substack{n_{1}+\cdots+n_{|t|}=n \\
n_{i} \geq 1}} \sum_{\substack{\Gamma_{i} \in T_{n_{i}, 2}, 2 \\
i=1, \ldots,|t|}} \sum_{\Gamma \in T_{n, 3}^{1}\left(t, \Gamma_{1}, \ldots, \Gamma_{|t|}\right)} W_{\Gamma_{\left(\{\overline{1}, \overline{2}\} \cup B_{\overline{3}}\right)}} W_{\Gamma_{/\{\overline{1}, \overline{2}\} \sqcup B_{\overline{3}}}} B_{\Gamma} \\
& =\sum_{n \geq 1} \frac{1}{n !} \sum_{\Gamma \in T_{n, 3}} W_{\Gamma_{\left(\{\overline{1}, \overline{2}\} \cup B_{\overline{3}}\right)}} W_{\Gamma_{/\{\overline{1}, \overline{2}\} \cup B_{\overline{3}}}} B_{\Gamma}
\end{aligned}
$$

which implies by Proposition 5 that $M_{n}^{1}(S)-M_{n}^{2}(S)=0$ for all $n>0$.

Uniqueness of the solution. We have seen that the perturbative SGA equations could be put at each order into the form $d S_{m}+H_{m}\left(S_{m-1}, \ldots, S_{1}\right)=0$, where the differential $d$ may be identified with the Hochschild differential on symbols.

Let $S$ and $S^{\prime}$ be two generating functions. By definition we have that $S_{1}=S_{1}^{\prime}=\alpha$. Now suppose that $S$ and $S^{\prime}$ are equal up to order $m-1$ (i.e., $S_{k}=S_{k}^{\prime}, k \leq m-1$ ). Thus $K_{m}:=S_{m}-S_{m}^{\prime} \in C^{\infty}\left(B_{2}\right)$ satisfies the following equation:

$$
d K_{m}=H_{m}\left(S_{1}, \ldots, S_{m-1}\right)-H_{m}\left(S_{1}^{\prime}, \ldots, S_{m-1}^{\prime}\right)=0 .
$$

As $H^{2}\left(C^{\infty}\left(B_{\bullet}\right), d\right)=V^{2}\left(\mathbb{R}^{d}\right)$ (bivector fields over $\mathbb{R}^{d}$ ) we have that $K_{m}$ can be written as $K_{m}=d k_{m}+\omega$, where $k_{m}$ is a 1-cochain and $\omega$ is a bivector field. Because of the homogeneity of $K_{m}$ in the $p$ 's we have that $\omega$ vanishes.

Claim. $k_{m}(p):=\frac{-1}{m+1} K_{m}^{1}(p, p)$ is a primitive of $K_{m}$, i.e., $d k_{m}=K_{m}$.

This claim proves the uniqueness because by assumption we have $K_{m}^{1}(p, p)=0$ which means that $k_{m}=0$ and thus $d k_{m}=K_{m}=0$. As for the claim, suppose that $K_{m}\left(p_{1}, p_{2}\right)=\sum_{|I|+|J|=m+1} K_{m}^{I, J} \frac{p_{1}^{I} p_{2}^{J}}{I ! J !}$, where we use the usual convention for the multi-indexes $I=\left(i_{1}, \ldots, i_{d}\right), J=\left(j_{1}, \ldots, j_{d}\right) \in \mathbb{N}^{d}$. Then an easy computation yields that

(1) $k_{m}(p)=\frac{-1}{m+1} K_{m}^{1}(p, p)=-\sum_{|I|=m+1} K_{m}^{e_{1}, I-e_{1}} \frac{p^{I}}{I !}$, where $e_{1}=(1,0, \ldots, 0)$,

(2) $d K_{m}=0$ implies that $K_{m}^{I, J}=K_{m}^{L, N}$ if $|I|+|J|=|L|+|N|$,

which implies that $d k_{m}\left(p_{1}, p_{2}\right)=K_{m}\left(p_{1}, p_{2}\right)$. 


\section{Comparison with Deformation Quantization}

In this section we make precise the statement that the generating function may be seen as the semi-classical approximation of the Kontsevich deformation formula. Namely Kontsevich gave in [13] an explicit formula for the associative deformation of the usual product of a function on $\mathbb{R}^{d}$ into the direction of a Poisson structure $\alpha$,

$$
f * g=f g+\sum_{n \geq 1} \frac{h^{n}}{n !} \sum_{\Gamma \in G_{n, 2}} W_{\Gamma} B_{\Gamma}(f, g),
$$

where $W_{\Gamma}$ are the weights and $B_{\Gamma}$ the bidifferential operators introduced in Sect. 5.

Definition 15. Consider a graph $\Gamma$ in $C_{n, 2}$, the set of connected graphs of type $(n, 2)$. We denote by $n_{\Gamma}:=\left|E_{\Gamma}^{a}\right|$ the number of aerial edges and $e_{\Gamma}:=\left|E_{\Gamma}^{g}\right|$ the number of ground edges.

In order to introduce the number of loops in a connected graph let us make the following remark. If $\Gamma$ is a connected graph of type $(n, 2)$ then $\Gamma$ must at least have $n-1$ aerial edges, which means that $n-1 \leq n_{\Gamma}$. On the other hand we have $n_{\Gamma}+e_{\Gamma}=2 n$. This implies that for connected Kontsevich graphs the number $n-e_{\Gamma}+1$ is always positive or zero.

Definition 16. For a connected graph of type $(n, 2)$ we call the number $n-e_{\Gamma}+1$ the number of loops of the graph and we denote it by $b_{\Gamma}$. We denote by $B_{n}^{l}$ the set of connected graphs of type $(n, 2)$ with l loops and we set $B^{l}=\cup_{n=1}^{\infty} B_{n}^{l}$. It is easy to see that $B_{n}^{0}$ are exactly the Kontsevich trees $T_{n, 2}$.

The following lemma shows that the star-product can be considered as a suitable exponentiation of a deformation of the Poisson structure.

Lemma 13 (Exponential formula). Let $f, g \in C^{\infty}(M)$. The star-product could be expressed as

$$
f * g(x)=\left.\exp \left(\frac{1}{h} D\left(h \partial_{x^{\prime}}, h \partial_{x^{\prime \prime}}, x\right)\right) f\left(x^{\prime}\right) g\left(x^{\prime \prime}\right)\right|_{x^{\prime}=x^{\prime \prime}=x}
$$

where $D\left(p_{1}, p_{2}, x\right)=\sum_{j=0}^{\infty} h^{j} D^{j}\left(p_{1}, p_{2}, x\right)$ and $D^{j}\left(p_{1}, p_{2}, x\right)=\sum_{\Gamma \in B^{j}} \frac{W_{\Gamma}}{|\Gamma| !}$ $\hat{B}_{\Gamma}\left(p_{1}, p_{2}, x\right)$.

Proof. By definition of the star-product, the definition of the $\hat{B}$ and using Lemma 9 of Sect. 5 we can do the following computation: 


$$
\begin{aligned}
& I=f * g(x) \\
& =\left.\left\{1+\sum_{n=1}^{\infty} \frac{h^{n}}{n !} \sum_{\Gamma \in G_{n, 2}} W_{\Gamma} \hat{B}_{\Gamma}\left(\partial_{x^{\prime}}, \partial_{x^{\prime \prime}}, x\right)\right\} f\left(x^{\prime}\right) g\left(x^{\prime \prime}\right)\right|_{x^{\prime}=x^{\prime \prime}=x} \\
& =\left.\left\{1+\sum_{n=1}^{\infty} \frac{h^{n}}{n !} \sum_{\substack{\Gamma \in G_{n, 2} \\
D(\Gamma)=\left(\Gamma_{1}, \ldots, \Gamma_{k}\right)}}\left(W_{\Gamma_{1}} \hat{B}_{\Gamma_{1}}\right) \ldots\left(W_{\Gamma_{k}} \hat{B}_{\Gamma_{k}}\right)\right\} f\left(x^{\prime}\right) g\left(x^{\prime \prime}\right)\right|_{x^{\prime}=x^{\prime \prime}=x} \\
& =\left\{1+\sum_{n=1}^{\infty} \frac{h^{n}}{n !} \sum_{k=1}^{n} \sum_{\substack{n_{1}, \ldots, n_{k} \in \mathbb{N} \backslash\{0\} \\
n_{1}+\cdots+n_{k}=n}} \sum_{\Gamma \in G_{n, 2}\left(n_{1}, \ldots, n_{k}\right)}\right. \\
& \left.\times\left(W_{\Gamma_{1}} \hat{B}_{\Gamma_{1}}\right) \ldots\left(W_{\Gamma_{k}} \hat{B}_{\Gamma_{k}}\right)\right\}\left.f\left(x^{\prime}\right) g\left(x^{\prime \prime}\right)\right|_{x^{\prime}=x^{\prime \prime}=x} \\
& =\left\{1+\sum_{n=1}^{\infty} \frac{h^{n}}{n !} \sum_{k=1}^{n} \sum_{\substack{n_{1}, \ldots, n_{k} \in \mathbb{N} \backslash\{0\} \\
n_{1}+\cdots+n_{k}=n}}\left(\frac{n !}{k ! n_{1} ! \ldots n_{k} !}\right)\right. \\
& \left.\times \sum_{\left(\Gamma_{1}, \ldots, \Gamma_{k}\right) \in C_{n_{1}, 2 \times \cdots \times C_{n_{k}, 2}}}\left(W_{\Gamma_{1}} \hat{B}_{\Gamma_{1}}\right) \ldots\left(W_{\Gamma_{k}} \hat{B}_{\Gamma_{k}}\right)\right\}\left.f\left(x^{\prime}\right) g\left(x^{\prime \prime}\right)\right|_{x^{\prime}=x^{\prime \prime}=x} \\
& =\left\{1+\sum_{k=1}^{\infty} \frac{1}{k !} \sum_{\substack{\left(\Gamma_{1}, \ldots, \Gamma_{k}\right) \in C_{n_{1}, 2 \times \cdots \times C_{n_{k}, 2}} \\
n_{1}, \ldots, n_{k} \in \mathbb{N} \backslash\{0\}}}\right. \\
& \left.\times\left(h^{n_{1}} \frac{W_{\Gamma_{1}}}{n_{1} !} \hat{B}_{\Gamma_{1}}\right) \ldots\left(h^{n_{k}} \frac{W_{\Gamma_{k}}}{n_{k} !} \hat{B}_{\Gamma_{k}}\right)\right\}\left.f\left(x^{\prime}\right) g\left(x^{\prime \prime}\right)\right|_{x^{\prime}=x^{\prime \prime}=x} \\
& =\left.\left\{1+\sum_{k=1}^{\infty} \frac{1}{k !}\left(\sum_{n=1}^{\infty} h^{n} \sum_{\Gamma \in C_{n, 2}} \frac{W_{\Gamma}}{n !} \hat{B}_{\Gamma}\right)^{k}\right\} f\left(x^{\prime}\right) g\left(x^{\prime \prime}\right)\right|_{x^{\prime}=x^{\prime \prime}=x} \\
& \left.=\exp \left\{\frac{1}{h} \sum_{n=1}^{\infty} h^{n+1} \sum_{\Gamma \in C_{n, 2}} \frac{W_{\Gamma}}{n !} \hat{B}_{\Gamma}\left(\partial_{x^{\prime}}, \partial_{x^{\prime \prime}}, x\right)\right)\right\}\left.f\left(x^{\prime}\right) g\left(x^{\prime \prime}\right)\right|_{x^{\prime}=x^{\prime \prime}=x} .
\end{aligned}
$$

Remarking that $\hat{B}_{\Gamma}\left(\partial_{x^{\prime}}, \partial_{x^{\prime \prime}}, x\right)=\frac{1}{h^{e} \Gamma} \hat{B}_{\Gamma}\left(h \partial_{x^{\prime}}, h \partial_{x^{\prime \prime}}, x\right)$, we can conclude that

$$
\begin{aligned}
f * g(x) & \left.=\exp \left\{\frac{1}{h} \sum_{n=1}^{\infty} \sum_{\Gamma \in C_{n, 2}} h^{n+1-e_{\Gamma}} \frac{W_{\Gamma}}{|\Gamma| !} \hat{B}_{\Gamma}\left(h \partial_{x^{\prime}}, h \partial_{x^{\prime \prime}}, x\right)\right)\right\} \\
& \left.=\exp \left\{\frac{1}{h} \sum_{j=0}^{\infty} h^{j} D^{j}\left(h \partial_{x^{\prime}}, h \partial_{x^{\prime \prime}}, x\right)\right)\right\} .
\end{aligned}
$$

The semi-classical part of the deformation formula is

$$
\frac{1}{h} D^{0}\left(h p_{1}, h p_{2}, x\right)
$$


It is easy to see that

$$
x\left(p_{1}+p_{2}\right)+\frac{1}{h} D^{0}\left(h p_{1}, h p_{2}, x\right)
$$

is exactly the formal symplectic groupoid generating function. It is in this sense that one can consider the generating function as a semi-classical approximation of the deformation formula.

We give now a quick but non-rigorous proof of the existence part of Theorem 1 . We use the technique of saddle point approximation (over non-really-well defined integrals). The following computations are then by no way a replacement of the rigorous and more technical argument developed in the previous sections.

First notice that as a consequence of the exponential formula of the previous lemma we have that

$$
e^{\frac{i}{\hbar} p_{1} x} * e^{\frac{i}{\hbar} p_{2} x}=e^{\frac{i}{h}\left(\sum_{j \geq 0}\left(\frac{\hbar}{i}\right)^{j} D^{j}\left(p_{1}, p_{2}, x\right)\right)} .
$$

We have replaced in the above identity the previously used formal parameter $h$ by $\frac{\hbar}{i}$ for better agreement with the notations in quantum mechanics. Moreover we have absorbed the term $x\left(p_{1}+p_{2}\right)$ into $D^{0}$. We keep using this convention through the following computation. Let us compute both sides of

$$
\underbrace{\left(e^{\frac{i}{\hbar} p_{1} x} * e^{\frac{i}{\hbar} p_{2} x}\right) * e^{\frac{i}{\hbar} p_{3} x}}_{A}=\underbrace{e^{\frac{i}{\hbar} p_{1} x} *\left(e^{\frac{i}{\hbar} p_{2} x} e^{\frac{i}{\hbar} p_{3} x}\right)}_{B}
$$

with the help of the asymptotical Fourier transform. We have then,

$$
A=(2 \pi \hbar)^{-d / 2} \int \hat{f}\left(p_{1}, p_{2}, p\right)\left(e^{\frac{i}{\hbar} p x} * e^{\frac{i}{\hbar} p_{3} x}\right) d p,
$$

where $\hat{f}\left(p_{1}, p_{2}, p\right)$ is the Fourier transform of

$$
f\left(p_{1}, p_{2}, x\right)=e^{\frac{i}{\hbar} p_{1} x} * e^{\frac{i}{\hbar} p_{2} x}=e^{\frac{i}{\hbar} \sum_{j=0}^{\infty}\left(\frac{\hbar}{i}\right)^{j} D^{j}\left(p_{1}, p_{2}, x\right)},
$$

that is,

$$
\hat{f}\left(p_{1}, p_{2}, p\right)=(2 \pi \hbar)^{-d / 2} \int e^{\frac{i}{\hbar}\left(\sum_{j=0}^{\infty}\left(\frac{\hbar}{i}\right)^{j} D^{j}\left(p_{1}, p_{2}, x\right)-p x\right)} d x .
$$

We use the method of the saddle point approximation to evaluate this integral when “ $\frac{\hbar}{i}$ is very small".

First notice that for functions of the form

$$
g_{\frac{\hbar}{i}}(x)=g_{0}(x)+\frac{\hbar}{i} g_{1}(x)+\left(\frac{\hbar}{i}\right)^{2} g_{2}(x)+\ldots
$$

a formal application of the implicit function theorem to $F\left(\frac{\hbar}{i}, x\right)=\nabla g_{\frac{\hbar}{i}}(x)$ tells us that

(1) $\exists \bar{x}: I \rightarrow \mathbb{R}^{n}$, where $I$ is a interval around zero so that $\bar{x}\left(\frac{\hbar}{i}\right)$ is an extremal point of $g_{\frac{\hbar}{i}}(x)$ if $\bar{x}(0)=\bar{x}$ is an extremal point of $g_{0}(x)$,

(2) $\bar{x}\left(\frac{\hbar}{i}\right)=\bar{x}-g_{0}^{-1^{\prime \prime}}(\bar{x}) g_{1}^{\prime}(\bar{x}) \frac{\hbar}{i}+\mathcal{O}\left(\left(\frac{\hbar}{i}\right)^{2}\right)$. 
Second, notice that we have the following asymptotical expansion:

$$
g_{\frac{\hbar}{i}}\left(\bar{x}\left(\frac{\hbar}{i}\right)\right)=g_{\frac{\hbar}{i}}\left(\left(\bar{x}-g_{0}^{-1^{\prime \prime}}(\bar{x}) g_{1}^{\prime}(\bar{x}) \frac{\hbar}{i}+\mathcal{O}\left(\left(\frac{\hbar}{i}\right)^{2}\right)\right) ;\right.
$$

around $\bar{x}$ we get

$$
g_{\frac{\hbar}{i}}\left(\bar{x}\left(\frac{\hbar}{i}\right)\right)=g_{0}(\bar{x})+\frac{\hbar}{i} g_{1}(\bar{x})+\mathcal{O}\left(\left(\frac{\hbar}{i}\right)^{2}\right) .
$$

Now if we apply the method of the stationary phase to

$$
I=\int e^{\frac{i}{\hbar} g_{\frac{\hbar}{i}}(x)} d x
$$

we find

$$
I \approx c\left(\bar{x}, \frac{\hbar}{i}\right) e^{\frac{i}{\hbar}\left(g_{0}(\bar{x})+\frac{\hbar}{i} g_{1}(\bar{x})\right)},
$$

where $\bar{x}$ is the extremal point of $g_{0}$.

Let us come back to the computation of $A$. With the preceding remarks in mind the computation of $\hat{f}\left(p_{1}, p_{2}, p\right)$ leads, through the application of the stationary phase method, to

$$
\hat{f}\left(p_{1}, p_{2}, p\right) \approx c\left(p_{1}, p_{2}, \bar{x}, \frac{\hbar}{i}\right) e^{\frac{i}{\hbar}\left(D^{0}\left(p_{1}, p_{2}, \bar{x}\right)-p \bar{x}+\frac{\hbar}{i} D^{1}\left(p_{1}, p_{2}, \bar{x}\right)\right)},
$$

where $c$ is a certain function of $p_{1}, p_{2}, \bar{x}$ and $\frac{\hbar}{i}$, and where $\bar{x}$, is a critical point of

$$
\left.D^{0}\left(p_{1}, p_{2}, x\right)-p x \quad \text { (i.e., } \quad \nabla_{x} D^{0}\left(p_{1}, p_{2}, \bar{x}\right)=p\right) .
$$

Then

$$
A \approx \int c\left(p_{1}, p_{2}, \bar{x}, \frac{\hbar}{i}\right) e^{\frac{i}{\hbar}\left(D^{0}\left(p_{1}, p_{2}, \bar{x}\right)-p \bar{x}+\frac{\hbar}{i} D^{1}\left(p_{1}, p_{2}, \bar{x}\right)+\sum_{j=0}^{\infty}\left(\frac{\hbar}{i}\right)^{j} D^{j}\left(p, p_{3}, x\right)\right)} d p .
$$

Using the same method as above again we obtain

$$
A \approx \tilde{C}\left(p_{1}, p_{2}, \bar{p}, \bar{x}, x, \frac{\hbar}{i}\right) e^{\frac{i}{\hbar}\left(D^{0}\left(p_{1}, p_{2}, \bar{x}\right)-\bar{p} \bar{x}+D^{0}\left(\bar{p}, p_{3}, x\right)\right)} e^{-D^{1}\left(\bar{p}, p_{3}, x\right)-D^{1}\left(p_{1}, p_{2}, \bar{x}\right)},
$$

where $\bar{x}$ is determined by $\nabla_{x} D^{0}\left(p_{1}, p_{2}, \bar{x}\right)=\bar{p}$ as above and $\bar{p}$ by $\nabla_{P_{1}} D^{0}\left(\bar{p}, p_{3}, x\right)=$ $\bar{x}$. Namely,

$$
\frac{d}{d p}\left[D^{0}\left(p_{1}, p_{2}, \bar{x}\right)-\bar{p} \bar{x}+D^{0}\left(\bar{p}, p_{3}, x\right)\right]=0
$$

gives

$$
\nabla_{x} D^{0}\left(p_{1}, p_{2}, \bar{x}\right) \frac{d \bar{x}}{d p}-\nabla_{x} D^{0}\left(p_{1}, p_{2}, \bar{x}\right) \frac{d \bar{x}}{d p}-\bar{x}+\nabla_{p_{1}} D^{0}\left(\bar{p}, p_{3}, x\right)=0 .
$$

By the same kind of computation we approximate $B$ for $\frac{\hbar}{i}$ "small enough",

$$
B \approx \tilde{C}\left(p_{2}, p_{3}, \tilde{p}, \tilde{x}, x, \frac{\hbar}{i}\right) e^{\frac{i}{\hbar}\left(D^{0}\left(p_{2}, p_{3}, \tilde{x}\right)-\tilde{p} \tilde{x}+D^{0}\left(p_{1}, \tilde{p}, x\right)\right)} e^{-D^{1}\left(p_{2}, p_{3}, \tilde{x}\right)-D^{1}\left(p_{1}, \tilde{p}, x\right)}
$$

with $\tilde{x}$ and $\tilde{p}$ determined by $\nabla_{x} D^{0}\left(p_{2}, p_{3}, \tilde{x}\right)=\tilde{p}$ and $\nabla_{p_{2}} D^{0}\left(p_{1}, \tilde{p}, x\right)=\tilde{x}$.

Equating $A$ and $B$ we then get that $D^{0}\left(p_{1}, p_{2}, x\right)$ satisfies the SGA equation. 


\section{References}

1. Bates, S., Weinstein, A.: Lectures on the geometry of quantization. Berkeley Mathematics Lecture Notes, 8. Providence, RI: and Berkeley, CA: American Mathematical Society, Berkeley Center for Pure and Applied Mathematics, 1997

2. Bayen, F., Flato, M., Fronsdal, C., Lichnerowicz, A., Sternheimer, D.: Deformation theory and quantization. I. Deformations of symplectic structures. Ann. Physics 111(1), 61-110 (1978)

3. Cattaneo, A.S.: The Lagrangian operad. Unpublished notes, http://www.math.unizh.ch/asc/ lagop.pdf

4. Cattaneo, A.S., Felder, G.: Poisson sigma models and deformation quantization. Euroconference on Brane New World and Noncommutative Geometry (Torino, 2000). Modern Phys. Lett. A 16(4-6), 179-189 (2001)

5. Cattaneo, A.S., Felder, G.: Poisson sigma models and symplectic groupoids. In: Quantization of singular symplectic quotients, Progr. Math. 198, Basel: Birkhäuser, 2001, pp. 61-93

6. Coste, A., Dazord, P., Weinstein, A.: Groupoïdes symplectiques. (French) [Symplectic groupoids] Publications du Département de Mathématiques. Nouvelle Série. A, Vol. 2, i-ii, Publ. Dép. Math. Nouvelle Sér. A, 87-2, Lyon: Univ. Claude-Bernard, 1987, pp. 1-62

7. Crainic, M.: Differentiable and algebroid cohomology, van Est isomorphisms, and characteristic classes. http://arxiv.org/math.DG/0008064, 2000

8. Crainic, M., Fernandes, R.L.: Integrability of Lie brackets. Ann. of Math. (2) 157(2), 575-620 (2003)

9. Hairer, E., Lubich, C., Wanner, G.: Geometric numerical integration. In: Structure-preserving algorithms for ordinary differential equations. Springer Series in Computational Mathematics, 31, Berlin: Springer-Verlag, 2002

10. Karabegov, K.: On Dequantization of Fedosov's Deformation Quantization. http://arxiv.org/ abs/math.QA/0307381, 2003

11. Karasëv, M.V.: Analogues of objects of the theory of Lie groups for nonlinear Poisson brackets. (Russian) Izv. Akad. Nauk SSSR Ser. Mat. 50(3), 508-538, 638 (1986)

12. Kathotia, V.: Kontsevich's universal formula for deformation quantization and the Campbell-BakerHausdorff formula. Internat. J. Math. 11(4), 523-551 (2000)

13. Kontsevich, M.: Deformation quantization of Poisson manifolds, I. Lett. Math. Phys. 66, 157-216 (2003)

14. Weinstein, A.: Symplectic groupoids and Poisson manifolds. Bull. Am. Math. Soc. (N.S.) 16(1), 101-104 (1987)

15. Weinstein, A., Xu, P.: Extensions of symplectic groupoids and quantization. J. Reine Angew. Math. 417, 159-189 (1991)

16. Weinstein, A.: Noncommutative geometry and geometric quantization. In: Symplectic geometry and mathematical physics (Aix-en-Provence, 1990), Progr. Math. 99, Boston, MA: Birkhäuser Boston, 1991, pp. 446-461

17. Weinstein, A.: Tangential deformation quantization and polarized symplectic groupoids. In: Deformation theory and symplectic geometry (Ascona, 1996), Math. Phys. Stud. 20, Dordrecht: Kluwer Acad. Publ. 1997, 301-314

18. Zakrzewski, S.: Quantum and classical pseudogroups. I. Union pseudogroups and their quantization. Commun. Math. Phys. 134(2), 347-370 (1990) 